\title{
Diatomáceas (Bacillariophyta) epilíticas da Bacia Hidrográfica do Rio Itajaí, Santa Catarina, Brasil'
}

\author{
Patricia Chiossi ${ }^{2 *}$ (D), Roselane Laudares Silva ${ }^{2}$ (D), Antonio Carlos Beaumord ${ }^{3}$ (i) \\ \& Lezilda Carvalho Torgan ${ }^{4}$
}

\author{
'Parte da dissertação de mestrado da primeira autora no Programa de Pós-Graduação em Biologia Vegetal \\ da Universidade Federal de Santa Catarina \\ 2Universidade Federal de Santa Catarina, Departamento de Botânica, Trindade, CEP 88040-970, Florianópolis, Santa Catarina, Brasil. \\ 3Universidade do Vale do Itajaí. Laboratório de Estudos de Impactos Ambientais. Caixa Postal 360, CEP 88302-202, Itajaí, \\ Santa Catarina, Brazil. \\ ${ }^{4}$ Secretaria do Meio Ambiente e Infraestrutura, Museu de Ciências Naturais. Rua Dr. Salvador França 1427, CEP 90690-000, \\ Porto Alegre, Rio Grande do Sul, Brazil. \\ *Autor para correspondência: patricia.chiossi@gmail.com
}

Recebido em 20.X.2020

Aceito em 05.IV.2021

DOI 10.21826/2446-82312021v76e2021007

\begin{abstract}
RESUMO - Este estudo objetivou conhecer a composição taxonômica das diatomáceas epilíticas das famílias Anomoeoneidaceae, Cymbellaceae, Gomphonemaceae, Achnanthidiaceae, Brachysiraceae e Naviculaceae e verificar a distribuição espacial dos táxons em 19 rios da Bacia Hidrográfica do Rio Itajaí. As amostragens abrangeram cinco sub-bacias, no período de abril a maio de 2009. Foram identificados 59 táxons específicos e infraespecíficos, distribuídos em 18 gêneros. As famílias mais representativas foram Naviculaceae e Gomphonemataceae com 15 táxons cada. Os gêneros mais ricos em espécies foram Gomphonema Ehrenberg (15 táxons) e Navicula Bory (13 táxons). São apresentados dados morfométricos e imagens em microscopia de luz e/ou eletrônica de varredura dos táxons. Um total de 28 táxons são citações pioneiras para o estado de Santa Catarina, vindo contribuir ao conhecimento florístico das diatomáceas em ambientes lóticos no sul do Brasil.
\end{abstract}

Palavras-chave: perifiton, sistema lótico, taxonomia, primeiros registros

\begin{abstract}
Epilithic diatoms (Bacillariophyta) from the Itajaí basin, Santa Catarina, Brazil. This study aimed to know the epilithic diatoms taxonomic composition of the Anomoeoneidaceae, Cymbellaceae, Gomphonemaceae, Achnanthidiaceae, Brachysiraceae and Naviculaceae families and to verify the spatial distribution of the taxa in 19 rivers of the Itajaí River Basin. The samplings covered five sub-basins, from April to May 2009. 59 specific and infra-specific taxa were identified, distributed in 18 genera. The most representative families were Naviculaceae and Gomphonemataceae with 15 taxa each. Gomphonema Ehrenberg (15 taxa) and Navicula Bory (13 taxa) were the richiest in species. Morphometric data and images are presented in light microscopy and / or scanning electron microscope. A total of 28 taxa are pioneering citations for the state of Santa Catarina, contributing to the floristic knowledge of diatoms in lotic environments in southern Brazil.
\end{abstract}

Keywords: periphyton, lotic system, taxonomy, first records

\section{INTRODUÇÃO}

As diatomáceas constituem um grupo de algas unicelulares, solitárias ou coloniais, muito abundante no plânkton e bentos de ambientes de água doce, onde fazem parte da biomassa algal e são os principais componentes da produtividade primária (Bellinger \& Singee 2010). Em rios, elas são encontradas preferencialmente aderidas a rochas e ao sedimento superficial e ocorrem onde a luz e nutrientes são suficientes para seu desenvolvimento. Características morfológicas como rafe, pedúnculos e almofadas mucilaginosas podem ser consideradas estratégias vantajosas para a fixação das diatomáceas em substratos e para a sua permanência em ambientes lóticos. As diatomáceas constituem uma das prevalescentes classe taxonômica em número de espécies, portanto os estudos de sua diversidade pode ser uma importante ferramenta para avaliação das condições ambientais (Butcher 1932, Stevenson 1996, Stevenson \& Pan 1999, Allan \& Castillo 2007).

O conhecimento taxonômico de diatomáceas em ambientes lóticos no Estado de Santa Catarina esteve concentrado na comunidade do plâncton. Estudos foram realizados no Rio Araranguá (Araújo et al. 1989), Rio Ratones (Fernandes et al. 1990), Rio Tubarão (Rodrigues \& Moreira-Filho 1990, Rodrigues 1984, 1988, 1991/1992) e no Rio Tavares (Felício-Fernandes et al. 1994, FelícioFernandes 1996). Investigações sobre a comunidade de algas epilíticas, neste estado, são escassos, podendo ser citados somente os estudos realizados por Burliga et al. (2005) e Sutil et al. (2007) no Rio Itajaí-Mirim, na mesma bacia do presente estudo. 
Este estudo teve como objetivo conhecer a composição taxonômica e a variação espacial das assembleias de diatomáceas epilíticas em 19 rios da Bacia Hidrográfica do Rio Itajaí, para dar suporte ao projeto "Delineamento de Sub-unidades de Ecorregiões Aquáticas: Costa Sul Brasileira" e ampliar o conhecimento da biodiversidade no estado de Santa Catarina.

\section{MATERIAL E MÉTODOS}

\section{Área de estudo}

A Bacia Hidrográfica do Rio Itajaí (Fig.1) situa-se, na região leste do Estado de Santa Catarina, na região Sul do Brasil. Apresenta uma área total de $15.000 \mathrm{~km}^{2}$, correspondendo a $16,15 \%$ do território catarinense e 25.000 $\mathrm{km}$ de cursos d'água.

A maioria dos rios desta bacia encontram-se em áreas rurais, já bem alteradas pela ação humana, recebendo efluentes de esgoto doméstico, sendo próximos às pastagens para criação de gado ou em áreas de reflorestamento de Pinus e Eucalyptus. As águas apresentam ph entre 7,0 e 7,9 , condutividade que varia entre 29,6 e $127,5 \mu{\mathrm{S} . \mathrm{cm}^{-1}}^{-}$, turbidez entre 0,45 e 38,26 UNT e DBO entre 0,19 e 1,80 mg.L ${ }^{-1}$ (Chiossi 2013).

\section{Amostragem e análise das amostas}

Foram realizadas amostragens em 19 rios, localizados em cinco sub-bacias da Bacia Hidrográfica do Rio Itajaí (Fig. 1, Tab. 1). As coletas foram realizadas em uma única ocasião, entre o final de abril e início de maio de 2009, sem presença de chuvas nas 24 horas anteriores à coleta. Foram coletadas aleatoriamente 10 rochas com aproximadamente $12 \mathrm{~cm}$ de diâmetro no leito rio a uma profundidade máxima de $30 \mathrm{~cm}$. As diatomáceas foram raspadas de uma área de $25 \mathrm{~cm}^{2}$ da superfície de cada rocha utilizando escovas de cerdas macias e jatos de água destilada. As amostras foram acondicionadas em frascos de polietileno de $250 \mathrm{ml}$ e fixadas com formalina. Uma alíquota de $10 \mathrm{ml} \mathrm{da}$ amostra foi oxidada com permanganato de potássio e ácido clorídrico de acordo com o método de Simonsen (1974) modificada por Moreira-Filho \& ValenteMoreira (1981). Foram montadas lâminas permanentes utilizando resina Naphrax. Foram observadas três lâminas de cada ponto. As medidas e fotografias foram realizadas utilizando microscópio óptico (MO) Leica DM 2500, com câmera de captura digital acoplada. Para a observação em microscopia eletrônica de varredura (MEV), o material foi montado em lamínula sobre fita de carbono em suportes de alumínio recobertos com ouro. Imagens da ultraestrutura das diatomáceas foram obtidas com microscópio JEOL$6390 \mathrm{LV}$, operando a $20 \mathrm{kV}$.
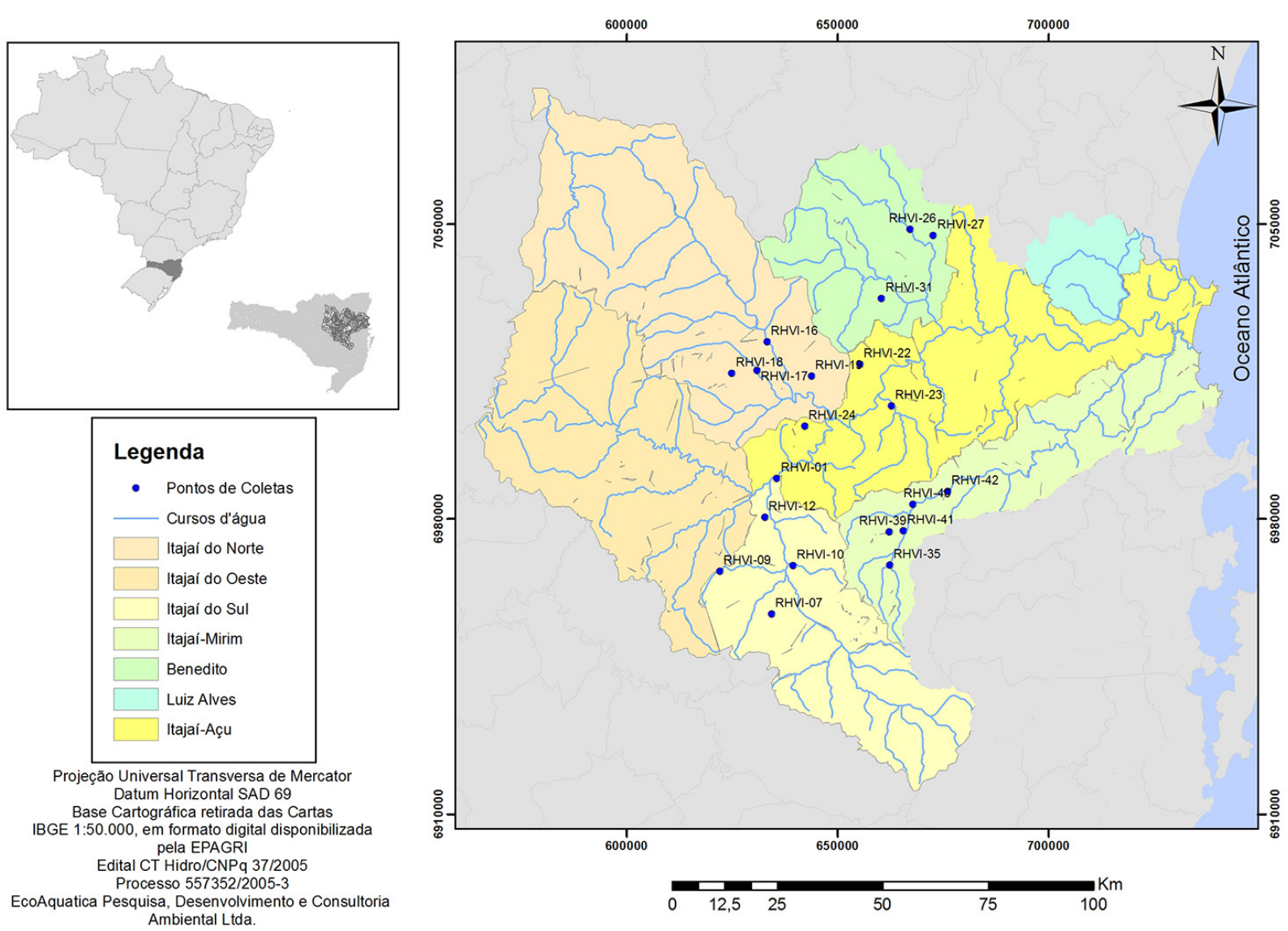

Figura 1. Mapa da localização da bacia hidrográfica do rio Itajaí, Santa Catarina, Brasil e a distribuição dos pontos amostrais nas sub-bacias estudadas. 
Tabela 1. Localização dos pontos amostrais na Bacia Hidrográfica do Rio Itajaí, Santa Catarina e referência do registro das lâminas depositadas no Herbário FLOR.

\begin{tabular}{|c|c|c|c|c|c|c|}
\hline Ponto & Longitude & Latitude & Município & Rio & Sub-bacia & FLOR \\
\hline 01 & $49^{\circ} 37^{\prime} 52^{\prime \prime} \mathrm{O}$ & $27^{\circ} 12^{\prime} 32 ” \mathrm{~S}$ & Laurentino & Ribeirão Albertina & Itajaí do Sul & 49737 \\
\hline 07 & $49^{\circ} 38^{\prime} 24^{\prime \prime} \mathrm{O}$ & $27^{\circ} 30^{\prime} 00^{\prime \prime S}$ & Petrolândia & Rio Antinha & Itajaí do Sul & 49743 \\
\hline 09 & $49^{\circ} 45^{\prime} 55^{\prime \prime} \mathrm{O}$ & $27^{\circ} 24^{\prime} 34^{\prime \prime} \mathrm{S}$ & Atalanta & Rio Dona Luiza & Itajaí do Sul & 49748 \\
\hline 10 & $49^{\circ} 35^{\prime} 27^{\prime \prime} \mathrm{O}$ & $27^{\circ} 23^{\prime} 46^{\prime \prime} \mathrm{S}$ & Ituporanga & Rio das Pedras & Itajaí do Sul & 50144 \\
\hline 12 & $49^{\circ} 39^{\prime} 34^{\prime \prime} \mathrm{O}$ & $27^{\circ} 17^{\prime} 36^{\prime \prime} \mathrm{S}$ & Ituporanga & Ribeirão Stoz & Itajaí do Sul & 50853 \\
\hline 16 & $49^{\circ} 39^{\prime} 55^{\prime \prime} \mathrm{O}$ & $26^{\circ} 55^{\prime} 00^{\prime \prime} \mathrm{S}$ & Pres. Getúlio & Ribeirão Ariribá & Itajaí do Norte & 50854 \\
\hline 17 & $49^{\circ} 40^{\prime} 56^{\prime \prime} \mathrm{O}$ & $26^{\circ} 58^{\prime} 43^{\prime \prime} \mathrm{S}$ & Withmarsum & Rio Arroio do Posto & Itajaí do Norte & 50855 \\
\hline 18 & $49^{\circ} 44^{\prime} 29^{\prime \prime} \mathrm{O}$ & $26^{\circ} 59^{\prime} 08^{\prime \prime} \mathrm{S}$ & Dona Emma & Rio Dona Emma & Itajaí do Norte & 50856 \\
\hline 19 & $49^{\circ} 33^{\prime} 05^{\prime \prime} \mathrm{O}$ & $26^{\circ} 59^{\prime} 22^{\prime \prime} \mathrm{S}$ & Ibirama & Rio Itajaí do Norte & Itajaí do Norte & 50857 \\
\hline 22 & $49^{\circ} 26^{\prime} 06^{\prime \prime} \mathrm{O}$ & $26^{\circ} 57^{\prime} 45^{\prime \prime} \mathrm{S}$ & Ascurra & Rio Itajaí-Açu & Itajaí-Açu & 50858 \\
\hline 23 & $49^{\circ} 21^{\prime} 36^{\prime \prime} \mathrm{O}$ & $27^{\circ} 03{ }^{\prime} 04^{\prime \prime} S$ & Apiúna & Ribeirão Neisse & Itajaí-Açu & 50859 \\
\hline 24 & $49^{\circ} 33^{\prime} 55^{\prime \prime} \mathrm{O}$ & $27^{\circ} 05^{\prime} 49^{\prime \prime} \mathrm{S}$ & Ibirama & Ribeirão das Pedras & Itajaí-Açu & 50860 \\
\hline 26 & $49^{\circ} 19^{\prime} 16^{\prime \prime} \mathrm{O}$ & $26^{\circ} 40^{\prime} 20^{\prime \prime} \mathrm{S}$ & Rio dos Cedros & Rio Esperança & Benedito & 50861 \\
\hline 27 & $49^{\circ} 15^{\prime} 53^{\prime \prime} \mathrm{O}$ & $26^{\circ} 41^{\prime} 04^{\prime \prime} \mathrm{S}$ & Rio dos Cedros & Rio Ada & Benedito & 50862 \\
\hline 31 & $49^{\circ} 23^{\prime} 10^{\prime \prime} \mathrm{O}$ & $26^{\circ} 49^{\prime} 19^{\prime \prime} \mathrm{S}$ & Benedito Novo & Ribeirão dos Russos & Benedito & 50863 \\
\hline 35 & $49^{\circ} 21^{\prime} 31^{\prime \prime} \mathrm{O}$ & $27^{\circ} 23^{\prime} 32^{\prime \prime} \mathrm{S}$ & Vidal Ramos & Rio Garrafão & Itajaí-Mirim & 50864 \\
\hline 39 & $49^{\circ} 21^{\prime} 38^{\prime \prime} \mathrm{O}$ & $27^{\circ} 19^{\prime} 16^{\prime \prime} \mathrm{S}$ & Pres. Nereu & Ribeirão Blink & Itajaí-Mirim & 50865 \\
\hline 40 & $49^{\circ} 18^{\prime} 16^{\prime \prime} \mathrm{O}$ & $27^{\circ} 15^{\prime} 41^{\prime \prime} \mathrm{S}$ & Pres. Nereu & Rio Indaial & Itajaí-Mirim & 50866 \\
\hline 41 & $49^{\circ} 19^{\prime} 38^{\prime \prime} \mathrm{O}$ & $27^{\circ} 19^{\prime} 04^{\prime \prime} \mathrm{S}$ & Pres. Nereu & Ribeirão Blink & Itajaí-Mirim & 50867 \\
\hline 42 & $49^{\circ} 13^{\prime} 19^{\prime \prime} \mathrm{O}$ & $27^{\circ} 13^{\prime} 56^{\prime \prime} \mathrm{S}$ & Botuverá & Rio Itupovão & Itajaí-Mirim & 50868 \\
\hline
\end{tabular}

O material analisado foi armazenado no laminário do herbário FLOR na Universidade Federal de Santa Catarina, Santa Catarina, Brasil (Tab. 1). A classificação taxonômica dos táxons seguiu o sistema proposto por Round et al. (1990) e modificado por Medlin \& Kaczmarska (2004).

\section{RESULTADOS E DISCUSSÃO}

A análise das amostras permitiu a determinação de 59 táxons específicos e infraespecíficos, distribuídos em 18 gêneros abaixo relacionados.

Bacillariophyta

Cymbellaceae Greville

Cymbella Agardh

Cymbella excisa var. angusta Krammer, Diatoms of Europe 3: 28, 159, pl. 9, figs. 8-18. 2002.

(Fig. 2)

Morfometria: compr. $39 \mu \mathrm{m}$, larg. $11,2 \mu \mathrm{m}, 11$ estrias em $10 \mu \mathrm{m}$ na margem dorsal e 10 estrias na margem ventral das valvas.

Material examinado: FLOR 49743.

Referências: Krammer (2002), Metzeltin et al. (2005).

Cymbella tumida (Brébisson ex Kützing) Van Heurck, Syn. Diat. Belg. Atlas. p. 64, pl. 2, fig. 10. 1880-1881.

(Figs. 3, 4)
Morfometria: compr. 50,5-75 $\mu \mathrm{m}$, larg. 16,0-20,6 $\mu \mathrm{m}, 10$ estrias em $10 \mu \mathrm{m}$ nas margens dorsais e ventrais das valvas.

Material examinado: FLOR 50860, 50864, 50866.

Referências: Krammer \& Lange-Bertalot (1986), Bes et al. (2012).

Cymbopleura (Krammer) Krammer

Cymbopleura amphicephala (Näegeli) Krammer, Diatoms of Europe 4: 70 pl. 91, fig. 1-18, pl. 93, fig. 2-8. 2003.

(Figs. 5, 6)

Morfometria: compr. 32,7-33,6 $\mu \mathrm{m}$, larg. 8,7-8,8 $\mu \mathrm{m}$, 11-12 estrias em $10 \mu \mathrm{m}$ na margem dorsal e 13-14 estrias na margem ventral das valvas.

Material examinado: FLOR 50853.

Referência: Krammer (2003).

Encyonema Kützing

Encyonema minutum (Hilse) Mann, Diatoms, p.667. 1990.

(Figs. 10, 11)

Morfometria: compr. 16,6-23,3 $\mu \mathrm{m}$, larg. 5,6-7,5 $\mu \mathrm{m}$, $11-12$ estrias em $10 \mu \mathrm{m}$ na margem dorsal e 11 estrias na margem ventral das valvas.

Material examinado: FLOR 49737, 50144, 50853, 50857 , 50858, 50859, 50860, 50864, 50865, 50866, 50867.

Referência: Bartozek et al. (2013). 
Encyonema cf. neogracile var. tenuipuntata Krammer, Biblioth. Diatomol. 36:143, pl. 83, figs. 4-7. 1997.

(Fig. 7)

Morfometria: compr. 48,8 $\mu \mathrm{m}$, larg. $8,7 \mu \mathrm{m} .12$ estrias 10 $\mu \mathrm{m}$ nas margens dorsal e ventral das valvas.

Material examinado: FLOR 50861.

Comentário: manteve-se a identificação em conferatum pelo fato do espécimen apresentar extremidades valvares não tão fortemente curvadas para a margem ventral, como ilustrado por Krammer (1997). As demais características conferem com a referida variedade.

Encyonema neomesianum Krammer, Biblioth. Diatomol. 36: 84, pl.40, fig.6-9, pl.54, fig.6-7, pl.99, fig.4-7. 1997.

\section{(Figs. 8, 9)}

Morfometria: compr. 47,7-55,4 $\mu \mathrm{m}$, larg. 12,3-12,4 $\mu \mathrm{m}$, 8-10 estrias em $10 \mu \mathrm{m}$ nas margens dorsais e ventrais das valvas.

Material examinado: FLOR 49737, 49743, 50853, 50856, 50859, 50860, 50864, 50866, 50867.

Referências: Krammer (1997), Bes et al. (2012).

Encyonema perpusillum (Cleve) Mann, Diatoms, p. 667. 1990.

(Figs. 12, 13)

Morfometria: compr. 15,7-17,1 $\mu \mathrm{m}$, larg. 4,78-4,97 $\mu \mathrm{m}$, 11 estrias em $10 \mu \mathrm{m}$ na margem dorsal e 10-11 estrias na margem ventral das valvas.

Material examinado: FLOR 49748, 50853, 50854, 50855 , 50856, 50857, 50858, 50859, 50860, 50861, 50862, 50866, 50867.

Referência: Bartozek et al. (2013).

Encyonema silesiacum (Bleisch) Mann, Diatoms, p. 667. 1990.

(Figs. 14-16)

Morfometria: compr. 28,3-33,2 $\mu \mathrm{m}$, larg. 6,7-10,4 $\mu \mathrm{m}$, 11-13 estrias em $10 \mu \mathrm{m}$ nas margens dorsais e ventrais das valvas.

Material examinado: FLOR 49737, 49743, 49748, 50144, 50853, 50854, 50855, 50856, 50859, 50860, 50861, 50862, 50863, 50864, 50865, 50866, 50867.

Referência: Bartozek et al. (2013).

\section{Encyonema sp.}

(Figs. 17-19)

Morfometria: compr. 17,3-31,8 $\mu \mathrm{m}$, larg. 6,9-8,6 $\mu \mathrm{m}$, 8-9 estrias em $10 \mu \mathrm{m}$ na margem dorsal e 10-13 estrias na margem ventral das valvas.

Material examinado: FLOR 50853, 50860.
Comentário: este táxon apresenta morfologia semelhante a Encyonema silesiacum (Bleisch) Mann, no entanto difere por apresentar estrias fortemente radiadas e em menor densidade na margem dorsal.

Placoneis Mereschkowsky

Placoneis gracilis Metzeltin, Lange-Bertalot \& GarcíaRodríguez, Iconogr. Diatomol. 15: pl.73, figs. 15-17. 2005.

(Fig. 22)

Morfometria: compr. 23,6 $\mu \mathrm{m}$, larg. 8,9 $\mu \mathrm{m}, 13$ estrias em $10 \mu \mathrm{m}$.

Material examinado: FLOR 50853.

Referência: Metzeltin et al. (2005).

Placoneis madagascariensis Lange-Bertalot \& Metzeltin, Iconogr. Diatomol. 11:54, pl. 27 figs. 37-40.2002.

(Figs. 20, 21)

Morfometria: compr. 18,7-23,2 $\mu \mathrm{m}$, larg. 7,3-8,0 $\mu \mathrm{m}$, 11-13 estrias em $10 \mu \mathrm{m}$.

Material examinado: FLOR 50144, 50862.

Referência: Metzeltin \& Lange-Betalot (2002).

Placoneis opportuna (Hustedt) Chudaev et Gololobova, Diatoms of the Glubokoe lake (Moscow region) p. 50, pl. 84, figs. 1-25. 2016.

(Fig. 24)

Morfometria: compr. 17,8 $\mu \mathrm{m}$, larg. $7,4 \mu \mathrm{m}, 14$ estrias em $10 \mu \mathrm{m}$.

Material examinado: FLOR 50853.

Referência: Chudaev \& Gololobova (2016).

Placoneis porifera (Hustedt) T. Ohtsuka \& Y. Fujita, Nova Hedwigia 73(1/2):107. 2001.

(Fig. 23)

Morfometria: compr. 29,4-40,9 $\mu \mathrm{m}$, larg. 14,8-15,6 $\mu \mathrm{m}$, 13-14 estrias em $10 \mu \mathrm{m}$.

Material examinado: FLOR 50857, 50863, 50866, 50867.

Referências: Hustedt (1944), Metzeltin \& Lange-Bertalot (1998), Ohtsuka Fujita (2001).

Gomphonemataceae

Gomphonema Ehrenberg

Gomphonema angustatum (Kützing) Rabenhorst, Fl. Europ. Alg. Aq. Dulc. Submar. Sect. I. p. 283, 1864.

(Figs. 25-27)

Morfometria: compr. 27,6-33,0 $\mu \mathrm{m}$, larg. 5,5-5,9 $\mu \mathrm{m}, 12-$ 14 estrias em $10 \mu \mathrm{m}$.

Material examinado: FLOR 50144, 50853, 50856, 50867.

Referências: Reichardt (1999), Tremarin et al. (2009). 

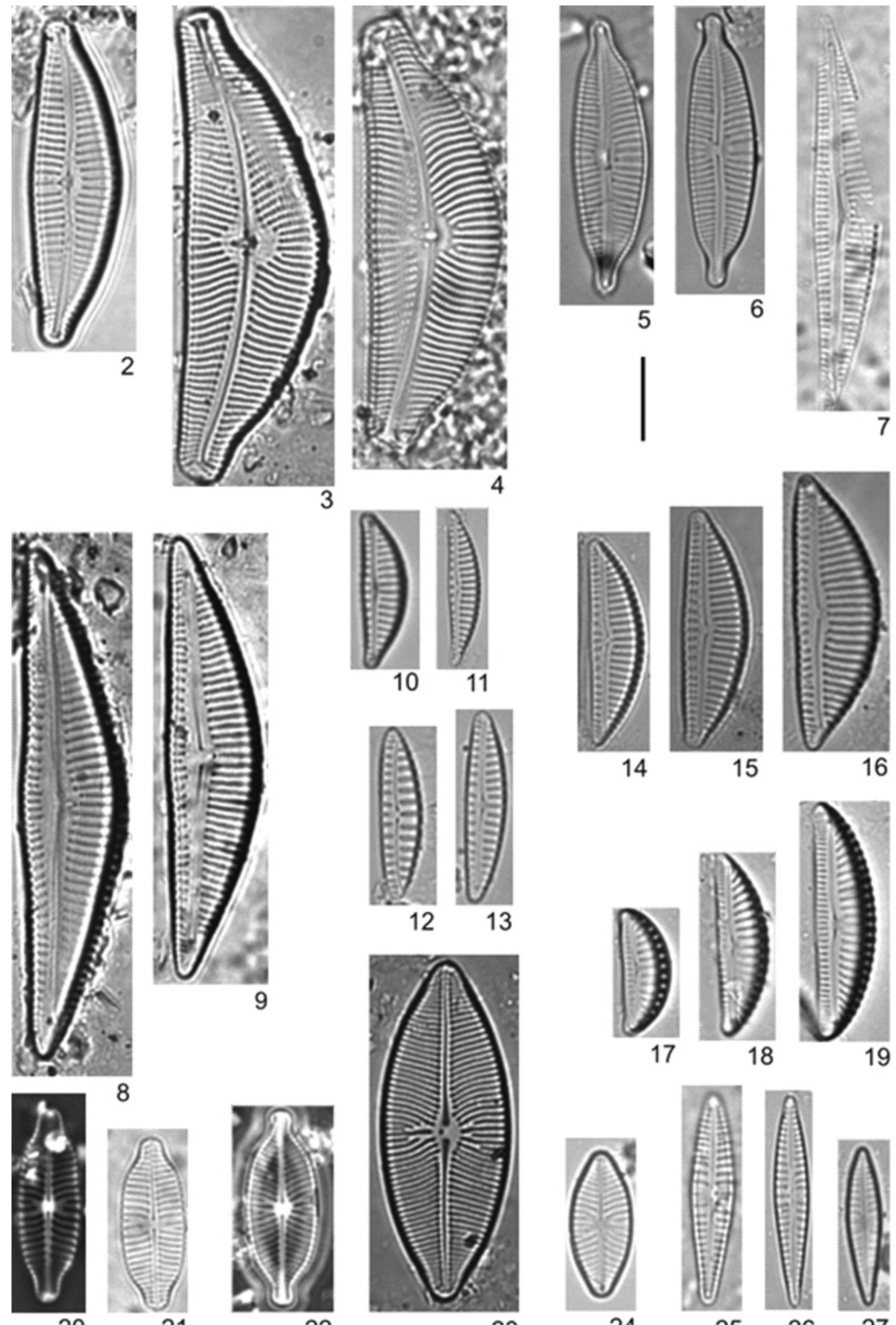

8
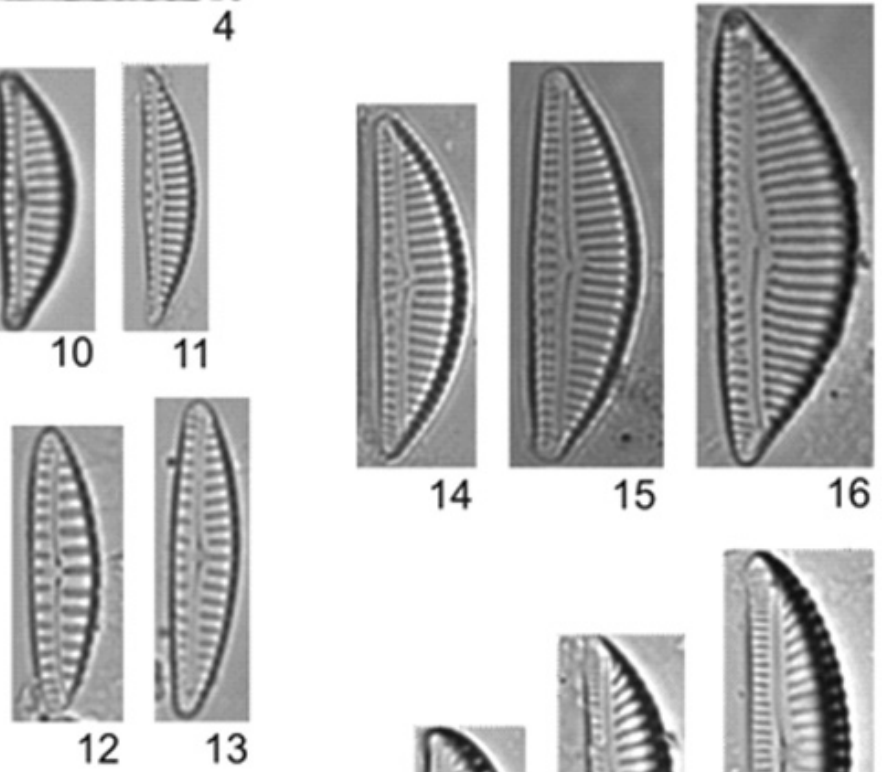

14

15

16
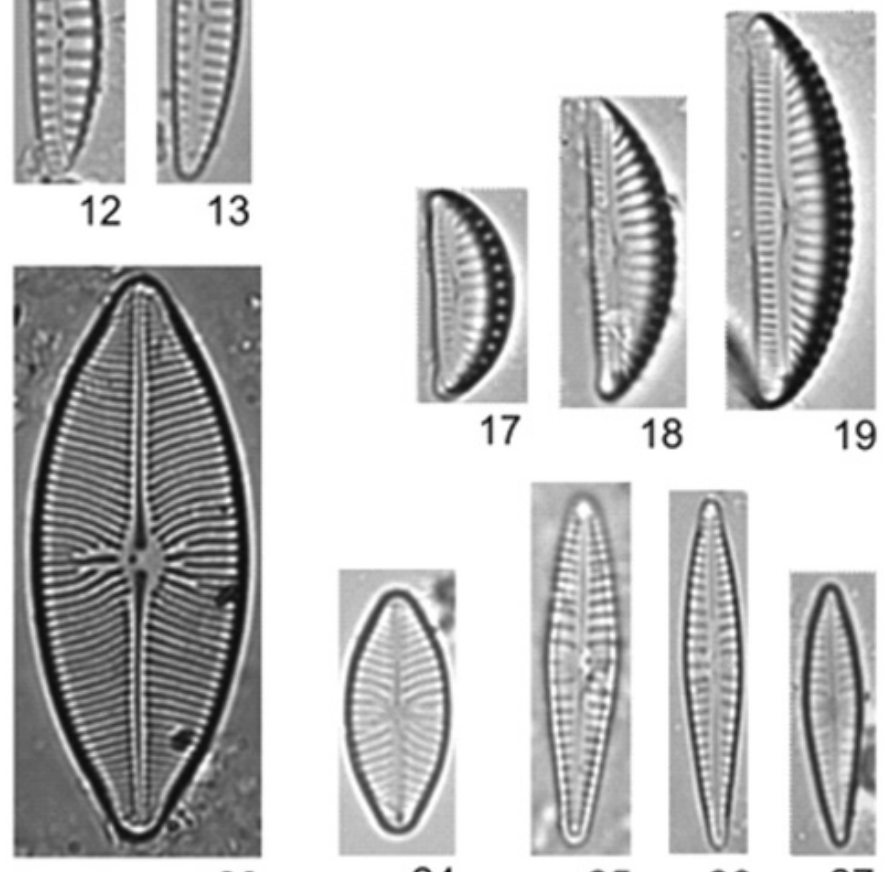

23
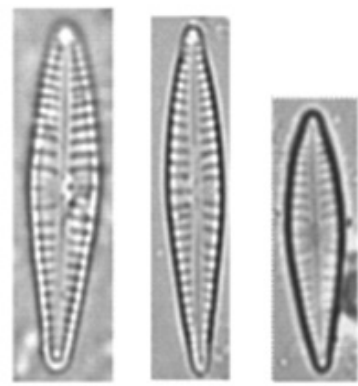

24

25

26

27

Figuras 2-27. Imagens em Microscópio Óptico. 2. Cymbella excisa var. angusta; 3, 4. C. tumida; 5, 6. Cymbopleura amphicephala; 7. Encyonema neogracile var. tenuipuctata; 8,9. E. neomesianum; 10, 11. E. minutum; 12, 13. E. perpusillum; 14-16. E. silesiacum; 17-19. Encyonema sp.; 20, 21. Placoneis madagascariensis; 22. Placoneis gracilis; 23. P. porifera; 24. P. opportuna; 25-27. Gomphonema angustatum. Barras $=10 \mu \mathrm{m}$ 
Gomphonema brasiliense ssp. pacificum G. Moser, LangeBertalot \&. Metzeltin, Bibl. Diatomol., 38:185-186; pl. 50, Fig. 1-6. 1998.

(Figs. 29, 48)

Morfometria: compr. 26-36,9 $\mu \mathrm{m}$, larg. 3,7 -53 $5 \mu \mathrm{m}, 12$ estrias em $10 \mu \mathrm{m}$.

Material examinado: FLOR 49748, 50144, 50857.

Referências: Moser et al. (1998), Medeiros et al. (2018).

Gomphonema brasiliensoide D. Metzeltin, Lange-Bertalot \& F. GarcíaRodríguez, Iconogr. Diatomol. 15: 80; pl. 149, Fig. 1-10. 2005

\section{(Figs. 28, 30)}

Morfometria: compr. 24,4-35,7 $\mu \mathrm{m}$, larg. 6,5-7,5 $\mu \mathrm{m}, 12-$ 13 estrias em $10 \mu \mathrm{m}$.

Material examinado: FLOR 49748, 50144, 50853, 50857, 50860, 50861, 50862.

Referências: Metzeltin et al. (2005), Medeiros et al. (2018).

Gomphonema gracile Ehrenberg, Infus. p. 27, pl. 8, fig. 3. 1838 .

(Figs. 33-36)

Morfometria: compr. 42,9-50,0 $\mu \mathrm{m}$, larg. 8,8-9,0 $\mu \mathrm{m}, 11$ 13 estrias em $10 \mu \mathrm{m}$.

Material examinado: FLOR 49748, 50854, 50856, 50858, 50861.

Referências: Patrick \& Reimer (1975), Tremarin et al. (2009).

Gomphonema incognitum Reichardt, Jüttner, Cox, Diatom Research, 19:245, figs. 33-35.

(Figs. 44-46)

Morfometria: compr. 18,4 - 22,6 $\mu \mathrm{m}$, larg. 3,8-4,5 $\mu \mathrm{m}$, 13-15 estrias em $10 \mu \mathrm{m}$.

Material examinado: FLOR 49748, 50144, 50853, 50854, 50857, 50858, 50859, 50860, 50861, 50862, 50863, 50865, 50866, 50868.

Referências: Jüttner et al. (2004), Reichardt (2005).

Gomphonema lagenula Kützing, Bacill. p. 85, pl. 30, fig. 60. 1844 .

(Figs. 37, 38)

Morfometria: compr. 18,4-30,7 $\mu \mathrm{m}$, larg. 6,3-7,5 $\mu \mathrm{m}, 12-$ 16 estrias em $10 \mu \mathrm{m}$.

Material examinado: FLOR 49737, 49743, 49748. 50144, 50853, 50854, 50855, 50856, 50859, 50860, 50861, 50862, 50864, 50865, 50866, 50867.

Referências: Tremarin et al. (2009), Medeiros et al. (2018).

Gomphonema mexicanum Grunow in van Heurck 1880: pl. 24 , fig. 3
(Figs. 51, 52)

Morfometria: compr. 36,2-44,4 $\mu \mathrm{m}$, larg. 9,4-11,2 $\mu \mathrm{m}$, 10-11 estrias em $10 \mu \mathrm{m}$.

Material examinado: FLOR 49748, 50862.

Referências: Van Heurck (1880-1881), Metzeltin et al. (2005).

Gomphonema parvulum (Kützing) Kützing, Spec. Alg. p. 65.1849 .

(Figs. 39, 40)

Morfometria: compr. 15,2-21,0 $\mu \mathrm{m}$, larg. 4,9-5,2 $\mu \mathrm{m}, 14-$ 16 estrias em $10 \mu \mathrm{m}$.

Material examinado: FLOR 49737, 49743, 49748, 50144, 50853, 50856, 50857, 50858, 50859, 50864, 50865, 50866, 50867.

Referências: Tremarin et al. (2009), Medeiros et al. (2018).

Gomphonema pseudoaugur Lange-Bertalot, Arch. Hydrobiol. 56(2):213-214, fig. 11-16, 79, 80, 1979.

(Figs. 49, 50)

Morfometria: compr. 32,4-44,4 $\mu \mathrm{m}$, larg. 9,3-10,5 $\mu \mathrm{m}$, 14-15 estrias em $10 \mu \mathrm{m}$.

Material examinado: FLOR 49743, 49748, 50853, 50857, 50859, 50867.

Referências: Krammer \& Lange-Bertalot (1986), Medeiros et al. (2018).

Gomphonema pumilum (Grunow) Reichardt \& LangeBertalot, Nova Hedwigia, 53(3-4): 528, pl. 6, fig. 4-11. 1991.

(Figs. 41-43)

Morfometria: compr. 13,4-15,0 $\mu \mathrm{m}$, larg. 3,9-4,5 $\mu \mathrm{m}, 11-$ 13 estrias em $10 \mu \mathrm{m}$.

Material examinado: FLOR 49743, 49748, 50854, 50856, 50862, 50868.

Referências: Reichardt \& Lange-Bertalot (1991), Medeiros et al. (2018).

Gomphonema rochense Metzeltin, Lange-Bertalot \& Garcia-Rodríguez, Iconogr. Diatomol. 15:87-88, pl. 145, figs. 23-27. 2005.

(Fig. 47)

Morfometria: compr. 33,1 $\mu \mathrm{m}$, larg. 7,1 $1 \mu \mathrm{m}, 13$ estrias em $10 \mu \mathrm{m}$.

Material examinado: FLOR 50862.

Referência: Metzeltin et al. (2005).

Gomphonema salae Lange-Bertalot \& Reichardt, Iconogr. Diatomol. 5: 124-125, pl. 157, fig. 3-5. 1998. 

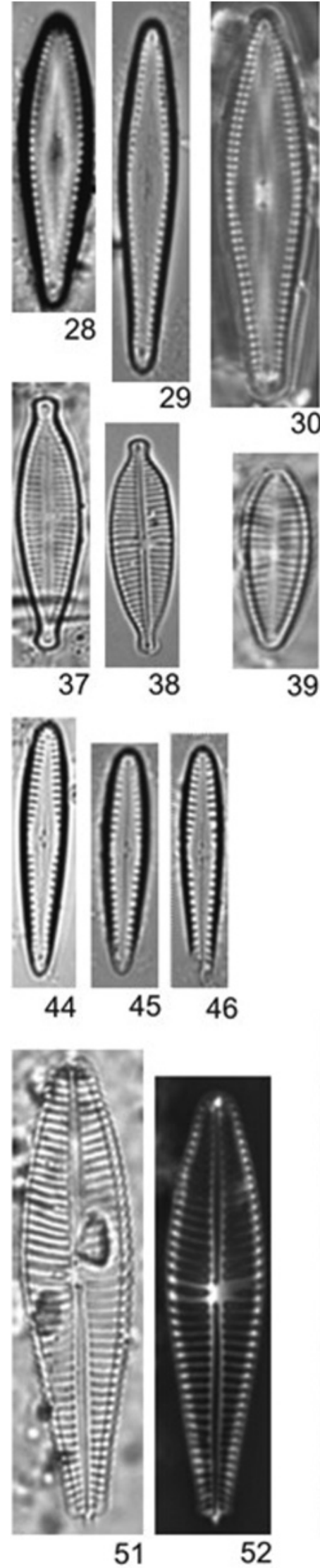

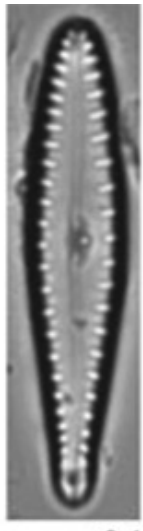

31
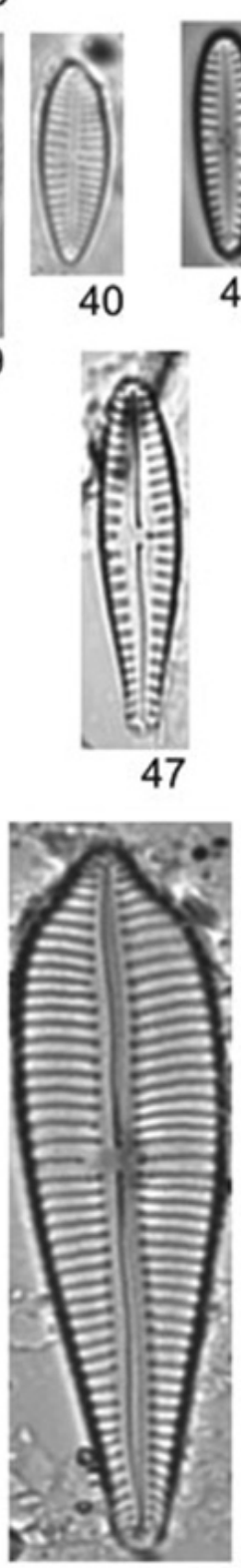

53
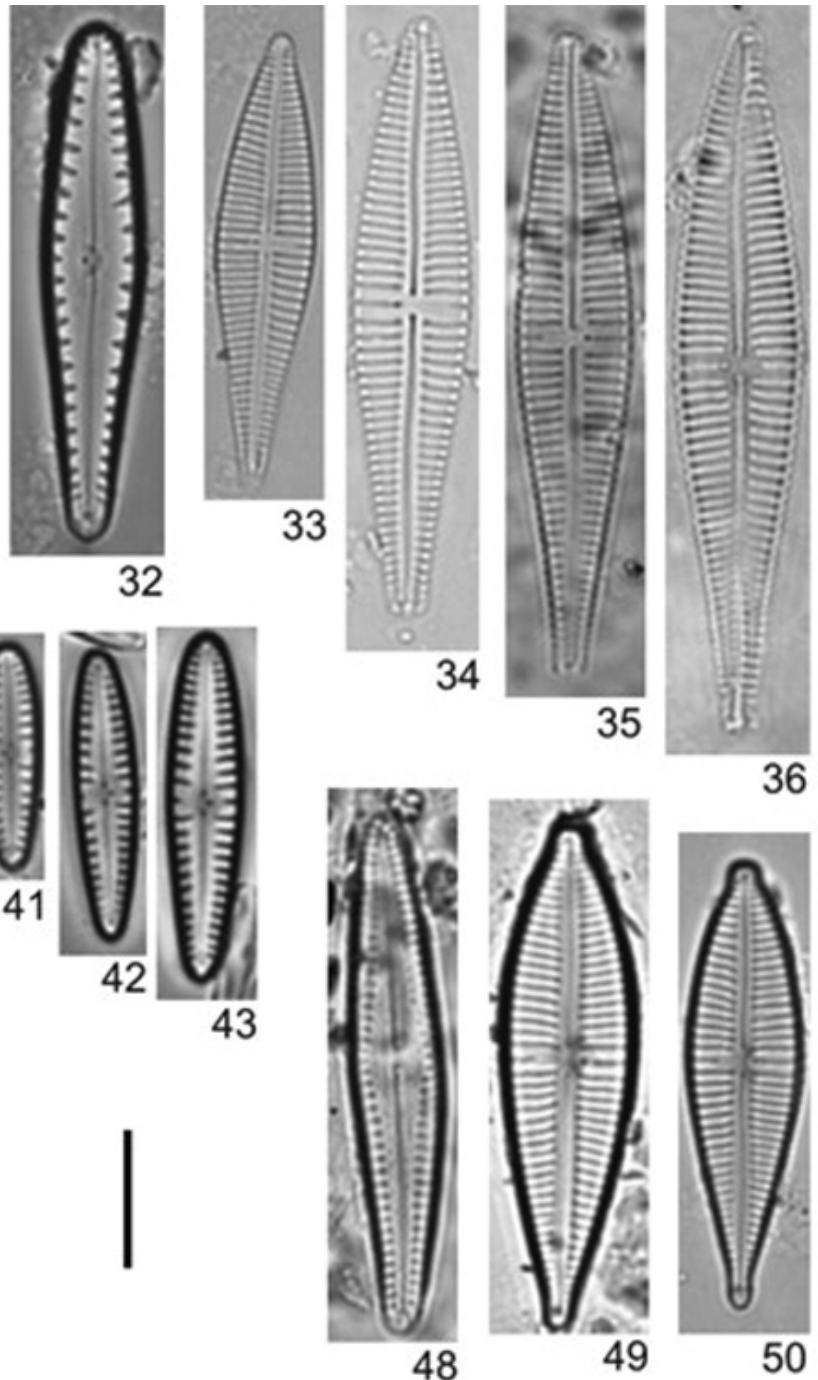

36
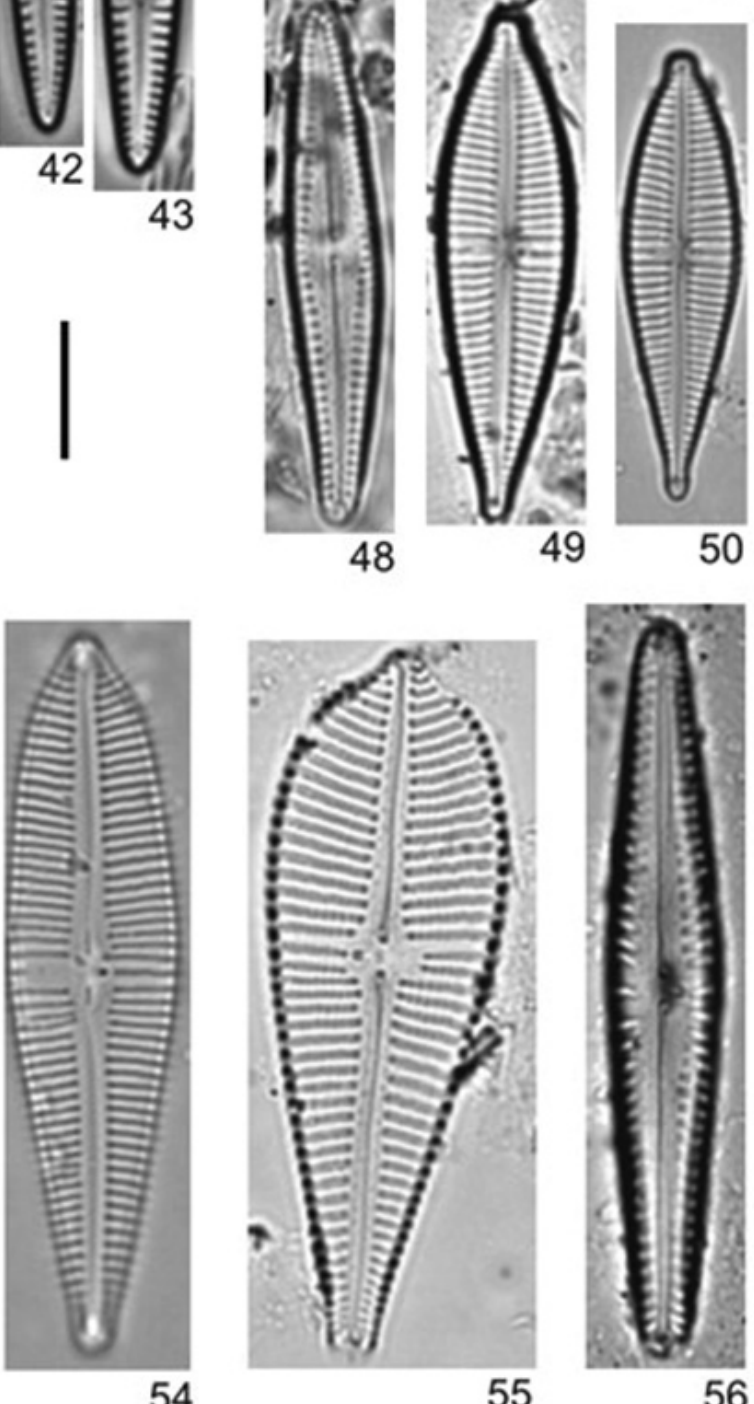

55

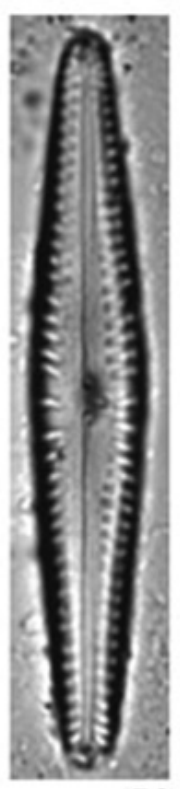

56

Figuras 28-56. Imagens em Microscópio Óptico. 28, 30. Gomphonema brasiliensoide; 29, 48. Gomphonema brasiliense ssp. pacificum; $\mathbf{3 1 ,} 32$. Gomphonema sp.1; 33-36. G. gracile; 37, 38. G. lagenula; 39, 40. G. parvulum; 41-43. G. pumilum; 44-46. Gomphonema incognitum; 47. Gomphonema rochense; 49, 50. G. pseudoaugur; 51, 52. G. mexicanum; 53, 55. G. turris var. coarctata; 54. G. salae; 56. Gomphonema sp. 2. Barras $=10 \mu \mathrm{m}$ 
(Fig. 54)

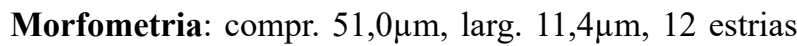
em $10 \mu \mathrm{m}$.

Material examinado: FLOR 50853.

Referência: Metzeltin \& Lange-Bertalot (1998).

Gomphonema turris var. coarctata (Frenguelli) Frenguelli, Rev. Mus. La Plata, Sec. Bot. 3:275. 1941.

(Figs. 53, 55)

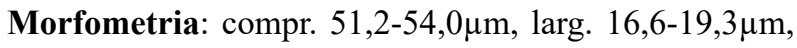
9-10 estrias em $10 \mu \mathrm{m}$ e 20 aréolas em $10 \mu \mathrm{m}$.

Material examinado: FLOR. 50856, 50861.

Referências: Sar et al. (2009), Tremarin et al. (2009).

\section{Gomphonema sp. 1}

(Figs. 31, 32)

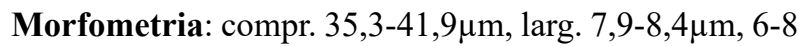
estrias em $10 \mu \mathrm{m}$.

Material examinado: FLOR 50853, 50856, 50867.

Comentário: os espécimens possuem esterno da rafe amplo, estigma localizado próximo ao nódulo central e estrias finas levemente convergentes em direção a área central. Essas caraterísticas não foram encontradas em espécies de Gomphonema apresentadas na literatura consultada.

\section{Gomphonema sp. 2}

(Fig. 56)

Morfometria: compr. 52,7 $\mu \mathrm{m}$, larg. $8,7 \mu \mathrm{m}, 9$ estrias em $10 \mu \mathrm{m}$.

\section{Material examinado: FLOR 50856.}

Comentário: o indivíduo apresenta morfometria semelhante a Gomphonema reimeri Kociolek \& J. C. Kingston (Kociolek \& Kingston 1999), porém difere por possuir ápices mais arredondados e estrias espaçadas na área central.

Achnanthidiaceae

Achnanthidium Kützing

Achnanthidium atomus (Hustedt) Monnier, Lange-Bertalot et Ector, Vie Milieu 54(2-3):133, Figs. 73-116, 124-130. 2004

(Figs. 57-59)

Morfometria: compr. 8,9-16,1 $\mu \mathrm{m}$, larg. 2,6-3,2 $\mu \mathrm{m}, 25$ estrias em $10 \mu \mathrm{m}$ na valva sem rafe, 30 estrias em $10 \mu \mathrm{m}$ na valva com rafe, 50 aréolas em $10 \mu \mathrm{m}$ na vista externa da valva com rafe.

Material examinado: FLOR 49737, 49743, 49748, 50144, $50853,50854,50855,50857,50858,50859,50860,50862$, 50864, 50865, 50866, 50867, 50868.
Referência: Monnier et al. (2004).

Achnanthidium exiguum (Grunow) Czarnecki, Mem. Cal. Acad. Sc. 17: 157. 1994.

(Figs. 60-63)

Morfometria: compr. 11,0-14,8 $\mu \mathrm{m}$, larg. 5,9-6,0 $\mu \mathrm{m}, 20-22$ estrias em $10 \mu \mathrm{m}$ na valva sem rafe e estrias inconspícuas na valva com rafe.

Material examinado: FLOR 49737, 49748, 50144, 50853, 50854, 50855, 50856, 50857, 50858, 50859, 50860, 50862, 50863, 50865, 50866, 50867, 50868.

Referência: Potapova (2010).

Achnanthidium minutissimum (Kützing) Czarnecki, Proc. $11^{\text {th }}$ Intern. Diat. Symp. 17: 157. 1994.

(Figs. 64-66)

Morfometria: compr. 12,8-14,8 $\mu \mathrm{m}$, larg. 3,0-3,3 $\mu \mathrm{m}, 33-35$ estrias em $10 \mu \mathrm{m}$ na valva sem rafe e 40 aréolas em $10 \mu \mathrm{m}$.

Material examinado: FLOR 49737, 49743, 49748, 50144, 50853, 50854, 50855, 50864, 50865, 50866, 50867, 50868.

Referência: Potapova \& Hamilton (2007).

Achnanthidium pyrenaicum (Hustedt) H. Kobayasi, Nova Hedwigia 65(1-4): 147-164. 1997.

(Figs. 67, 68)

Morfometria: compr. 11,8-12,1 $\mu \mathrm{m}$, larg. 3,9-4,4 $\mu \mathrm{m}, 22$ estrias em $10 \mu \mathrm{m}$ na valva com rafe; 24 estrias em $10 \mu \mathrm{m}$ na valva sem rafe.

Material examinado: FLOR 49737, 50853, 50864, 50865, 50867.

Referência: Potapova \& Ponader (2004).

Lemnicola Round \& Basson

Lemnicola hungarica (Grunow) Round \& Basson, Diatom Research 12(1): 77, fig. 26-31. 1997.

(Figs. 69, 70)

Morfometria: compr. 20,4-28,7 $\mu \mathrm{m}$, larg. 7,6-8, $0 \mu \mathrm{m}, 20$ estrias em $10 \mu \mathrm{m}$ na valva sem rafe.

Material examinado: FLOR 50857, 50859, 50866, 50867.

Referência: Round \& Basson (1997).

Planothidium Round \& Bukhtiyarova

Planothidium bagualensis Wetzel \& Ector, Phytotaxa 156(4): 203, figs. 2-19. 2014.

(Figs. 75-79)

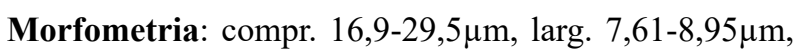
11 estrias em $10 \mu \mathrm{m}$ na valva com rafe e na valva sem rafe. 

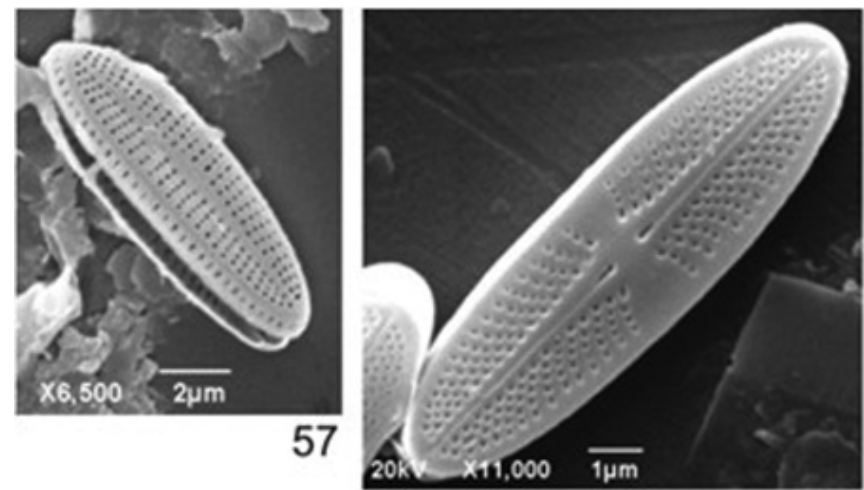

58

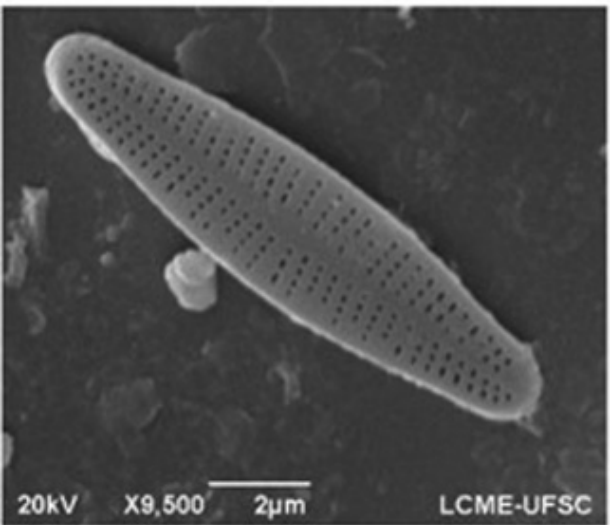

64

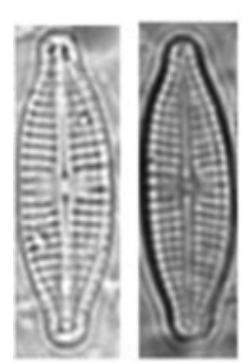

71

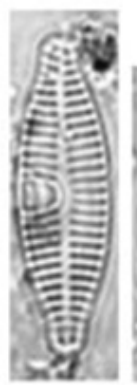

73

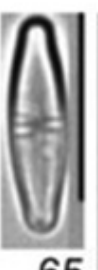

65

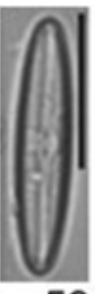

59

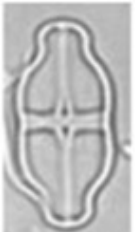

60

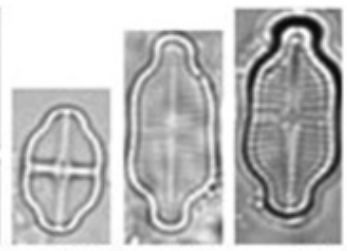

61

62

63

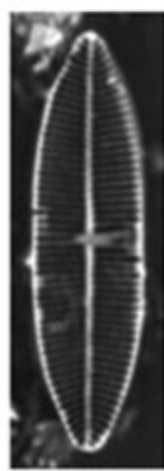

69
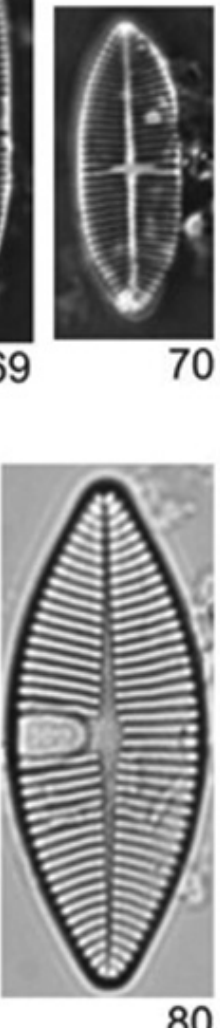
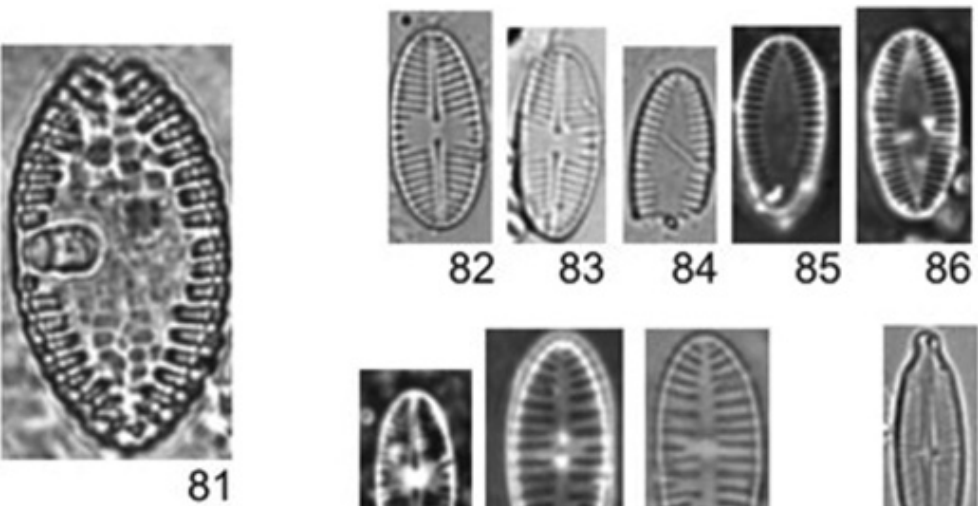

77

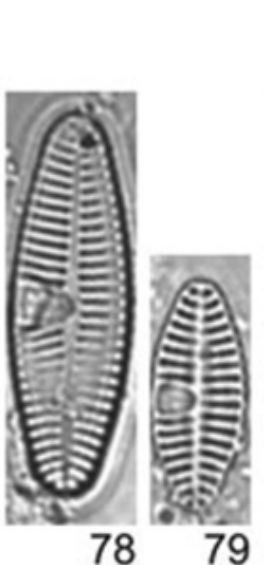

82

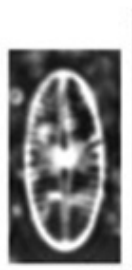

87

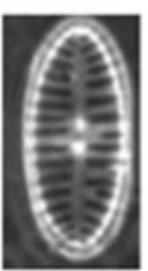

88

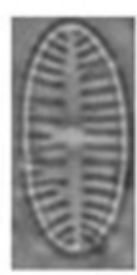

89
85

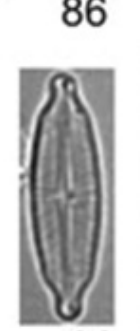

90
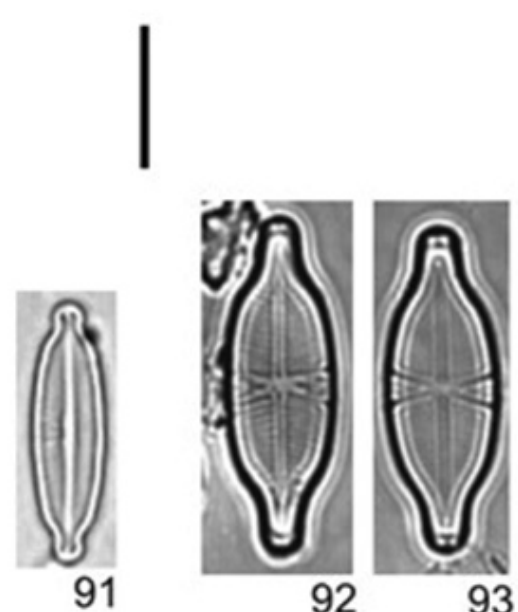

Figuras 57-93. Imagens em Microscópio Óptico e em Microscópio Eletrônico de Varredura. Achnanthidium atomus; 57, 58. Vista externa em MEV; 59. Vista valvar em MO; 60-63. A. exiguum; 64-66. A. minutissimum; 64. Vista externa (MEV); 65, 66. MO; 67, 68. A. pyrenaicum; 69, 70. Lemnicola hungarica; 71-74. Planothidium incuriatum; 75-79. P. bagualensis; 80. P. heteromorphum; 81. P. salvadorianum; 82-86. Psammothidium hustedtii; 87-89. Platessa oblongella; 90, 91. Adlafia drouetiana; 92, 93. Capartogramma crucicula. Barras $=10 \mu \mathrm{m}$ 
Material examinado: FLOR 49737, 49748, 50144, 50853, 50854, 50855, 50857, 50859, 50860, 50861, 50863, 50865, 50866, 50867.

Referência: Wetzel \& Ector (2014).

Planothidium heteromorphum (Grunow) Lange-Bertalot, Iconogr. Diatomol. 6: 282. 1999.

(Fig. 80)

Morfometria: compr. 35,4 $\mu \mathrm{m}$, larg. 14,5 $\mu \mathrm{m}, 13$ estrias em $10 \mu \mathrm{m}$ na valva sem rafe.

Material examinado: FLOR 50855.

Referências: Metzeltin et al. (2005), Bartozek et al. (2013).

Planothidium incuriatum Wetzel, Van de Vijver \& Ector, Phytotaxa 138(1):43-57. 2013.

(Figs. 71-74)

Morfometria: compr. 19,1-22,3 $\mu \mathrm{m}$, larg. 6,9-7,4 $\mu \mathrm{m}, 13-14$ estrias em $10 \mu \mathrm{m}$ na valva com rafe e na valva sem rafe.

Material examinado: FLOR 49737, 49743, 49748, 50144, 50854, 50855, 50856, 50857, 50858, 50859, 50860, 50862, 50864, 50865, 50866, 50867.

Referências: Wetzel et al. (2013), Silva et al. (2017).

Planothidium salvadorianum (Hustedt) Lange-Bertalot, Iconogr. Diatomol. 6:285. 1999.

(Fig. 81)

Morfometria: compr. 27,8 $\mu \mathrm{m}$, larg. $15,89 \mu \mathrm{m}, 7$ estrias em $10 \mu \mathrm{m}$ na valva sem rafe.

Material examinado: FLOR 50865.

Referência: Ferrari \& Ludwig (2007).

Platessa Lange-Bertalot

Platessa oblongella (Østrup) C.E. Wetzel, Nova Hedwigia Beiheft 146: 213, figs. 33-43. 2017.

(Figs. 87-89)

Morfometria: compr. 16,1 $\mu \mathrm{m}$, larg. 7,7 $\mu \mathrm{m}, 18-20$ estrias em $10 \mu \mathrm{m}$ na valva com rafe, $10-16$ estrias em $10 \mu \mathrm{m}$ na valva sem rafe.

Material examinado: FLOR 50858, 50865.

Referência: Wetzel et al. (2017).

Psammothidium Buhtkiyarova \& Round

Psammothidium hustedtii (Krasske) S. Mayama, Diatom 18:90. 2002.

(Figs. 82-86)

Morfometria: compr. 13,4-14,3 $\mu \mathrm{m}$, larg. 6,2-6,3 $\mu \mathrm{m}, 17$ estrias em $10 \mu \mathrm{m}$ na valva sem rafe.

Material examinado: FLOR 49737, 50853, 50857, 50867.
Referência: como Achnanthes hustedtii (Krasske) Reimer (Patrick \& Reimer 1966).

Anomoeoneidaceae D. G. Mann

Adlafia Moser, Lange-Bertalot \& Metzeltin

Adlafia drouetiana (Patrick) Metzeltin \& Lange-Bertalot, Iconogr. Diatomol. 5: 21, pl. 86, fig. 14-19. 1998.

(Figs. 90, 91)

Morfometria: compr. 13,9-16,9 $\mu \mathrm{m}$, larg. 4,6-5, $1 \mu \mathrm{m}, 24-$ 26 estrias em $10 \mu \mathrm{m}$.

Material examinado: FLOR 49743, 50854, 50855, 50856, 50857, 50858, 50859, 50864, 50866, 50867, 50868.

Referências: Metzeltin \& Lange-Bertalot (1998), Bes, et al. (2012), Silva et al. (2017).

Cymbellaceae Greville

Geissleria Lange-Bertalot e Metzeltin

Geissleria aikenensis (Patrick) Torgan \& Oliveira, in Proceedings of 16th Inter. Diat. Symp., p. 116, Figs. 6-19. 2001.

(Figs. 94, 95)

Morfometria: compr. 15,7-24,1 $\mu \mathrm{m}$, larg. 6,0-7,4 $\mu \mathrm{m}, 14-$ 16 estrias em $10 \mu \mathrm{m}$.

Material examinado: FLOR 49737, 49743, 501449, 50144, 50853, 50854, 50855, 50856, 50857, 50858, 50859, 50860, 50862, 50864, 50865, 50866, 50867, 50868.

Referência: Torgan \& Oliveira (2001).

Naviculaceae Kützing

Capartogramma Kufferath

Capartogramma crucicula (Grunow ex Cleve) Ross, Bull. British. Mus. 3(2): 59-64, pl. 1B, fig. 1a, 8-11. 1963.

(Figs. 92, 93)

Morfometria: compr. 20,6-28,6 $\mu \mathrm{m}$, larg. 8,0-9,2 $\mu \mathrm{m}, 20$ 22 estrias em $10 \mu \mathrm{m}$.

Material examinado: FLOR 49743, 50144, 50853, 50854, 50856.

Referências: Ross (1963), Silva et al. (2017).

Hippodonta Lange-Bertalot, Metzeltin \& Witkowski

Hippodonta hungarica (Grunow) Lange-Bertalot, Metzeltin \& Witkowski, Iconograph. Diatomol. 4: 259, pl. 1, fig. 22-26. 1996.

(Figs. 100, 101)

Morfometria: compr. 19,0-21,3 $\mu \mathrm{m}$, larg. 5,0-5,8 $\mu \mathrm{m}, 10$ 11 estrias em $10 \mu \mathrm{m}$.

Material examinado: FLOR 49737, 50144, 50853.

Referência: Lange-Bertalot, Metzeltin \& Witkowski (1996). 
Navicula Bory

Navicula cryptocephala Kützing, Bacill. p.95, pl. 3, fig. 20-26. 1844.

(Figs. 104, 105)

Morfometria: compr. 24,0-35,4 $\mu \mathrm{m}$, larg. 4,9-6,0 $\mu \mathrm{m}, 15-$ 17 estrias em $10 \mu \mathrm{m}$.

Material examinado: FLOR 49737, 49748, 50855, 50866.

Referências: Patrick \& Reimer (1966), Lange-Bertalot \& Metzeltin (1996), Bes et al. (2012).

Navicula cryptotenella Lange-Bertalot, Biblioth. Diatomol. 9: 62, pl.18, fig. 22-23, pl.19, fig.1-10, pl.27, fig.1. 1985.

(Figs. 106, 107)

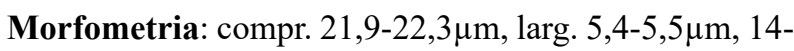
16 estrias em $10 \mu \mathrm{m}$.

Material examinado: FLOR 49737, 49743, 49748, 50144, 50853, 50854, 50855, 50857, 50858, 50859, 50864, 50866, 50867, 50868.

Referências: Lange-Bertalot \& Metzeltin (1996), Metzeltin \& García-Rodrigues (2003).

Navicula eichhorniaephila Manguin ex Kociolek \& Reviers, Cryptog. Algol. 17(3), pl. 44, figs 1-6. 1996.

(Figs. 122, 123)

Morfometria: compr. 30,7-34,4 $\mu \mathrm{m}$, larg. 5,8-6,1 $\mu \mathrm{m}, 12-$ 15 estrias em $10 \mu \mathrm{m}$.

Material examinado: FLOR 50856.

Referência: Rumrich et al. (2000).

Navicula gregaria Donkin, Quart. Jour. Micr. Sc. 1: 10, pl. 1, fig. 10. 1861.

\section{(Fig. 110)}

Morfometria: compr. 18,7-25,6 $\mu \mathrm{m}$, larg. 5,5-6,1 $\mu \mathrm{m}, 15-$ 18 estrias em $10 \mu \mathrm{m}$.

Material examinado: FLOR 49748, 50854, 50856, 50857, 50858, 50866, 50867, 50868.

Referências: Krammer \& Lange-Bertalot (1986), Bes et al. (2012).

Navicula leptostriata Jørgensen, Kong. Danske Vidensk. Selsk., Biologiske Shrifter. 5 (2): 59; pl. 2, fig. 25. 1948.

(Figs. 102, 103)

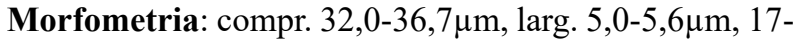
18 estrias em $10 \mu \mathrm{m}$.

Material examinado: FLOR 50853, 50859, 50865.

Referência: Krammer \& Lange-Bertalot (1991).

Navicula microdigitoradiata Lange-Bertalot, Biblioth. Diatomol. 27: 123, pl. 58, fig. 9-15, pl. 59, fig. 23-24. 1993.
(Figs. 111, 112)

Morfometria: compr. 15,9-18,3 $\mu \mathrm{m}$, larg. 4,3-4,4 $\mu \mathrm{m}, 18$ estrias em $10 \mu \mathrm{m}$.

Material examinado: FLOR 49748, 50853, 50859, 50867.

Referência: Lange-Bertalot (1993).

Navicula namibica Lange-Bertalot \& Rumrich, Biblioth. Diatomol. 27: 124, pl. 58, fig. 16-24. 1993.

(Figs. 124, 125)

Morfometria: compr. 28,7-30,3 $\mu \mathrm{m}$, larg. 5,8-6,4 $\mu \mathrm{m}, 15$ estrias em $10 \mu \mathrm{m}$.

Material examinado: FLOR 50853, 50859.

Referência: Lange-Bertalot (1993).

Navicula notha Wallace, Notulae Naturae. 331: 4, pl. 1, fig. 4 A-D. 1960.

(Figs. 108, 109)

Morfometria: compr. 28,9-30,8 $\mu \mathrm{m}$, larg. 5,2-6,0 $\mu \mathrm{m}, 15-$ 17 estrias em $10 \mu \mathrm{m}$.

Material examinado: FLOR 49737, 49743, 49748, 50144, 50853, 50857, 50858, 50859, 50864, 50866, 50867.

Referências: Lange-Bertalot \& Metzeltin (1996), Bes et al. (2012).

Navicula rostellata Kützing, Kies. Bacill. Diat. p. 95, pl. 3, fig. 65. 1844.

(Figs. 118, 119)

Morfometria: compr. 35,8-43,1 $\mu \mathrm{m}$, larg. $8,6-9,2 \mu \mathrm{m}, 12-$ 15 estrias em $10 \mu \mathrm{m}$.

Material examinado: FLOR 49743, 49748, 50144, 50853, 50854, 50855, 50856, 50857, 50858, 50859, 50862, 50864, 50866, 50867.

Referências: Metzeltin \& García-Rodrigues (2003), Bes et al. (2012).

Navicula salinicola Hustedt, Abh. Natuw. Ver. Bremen. 31(3): 638, Fig. 61-69. 1939.

(Figs. 113-115)

Morfometria: compr. 14,4-17,4 $\mu \mathrm{m}$, larg. 3,7-3,8 $\mu \mathrm{m}, 18$ estrias em $10 \mu \mathrm{m}$.

Material examinado: FLOR 50853.

Referências: Hustedt (1939), Krammer \& Lange-Bertalot (1986).

Navicula symmetrica Patrick, Bol. Mus. Nac. 2: 5, fig. 6. 1944.

(Figs. 120, 121)

Morfometria: compr. 34,0-35,3 $\mu \mathrm{m}$, larg. 6,8-7,6 $\mu \mathrm{m}, 13-$ 15 estrias em $10 \mu \mathrm{m}$. 


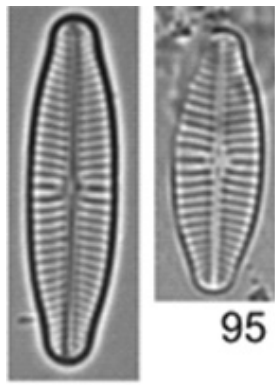

94
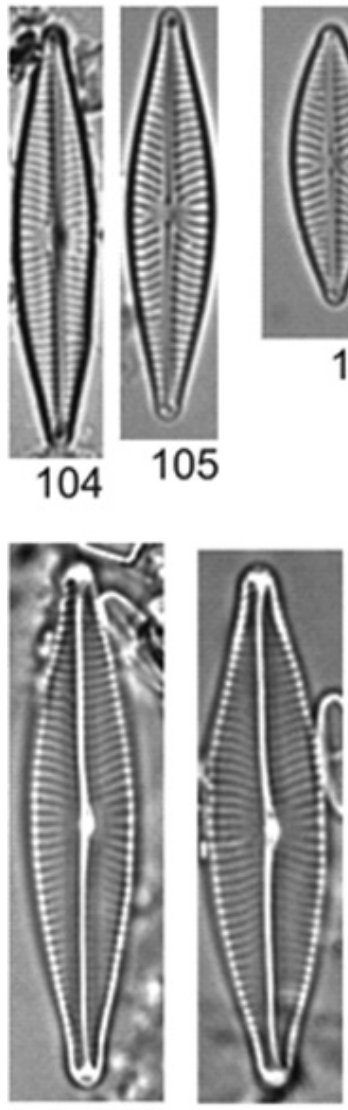

116
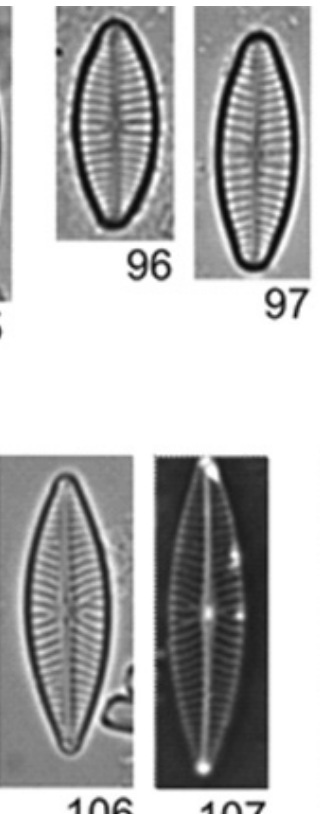

106
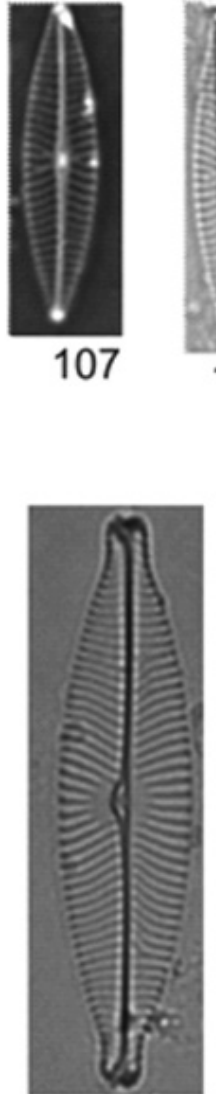

118
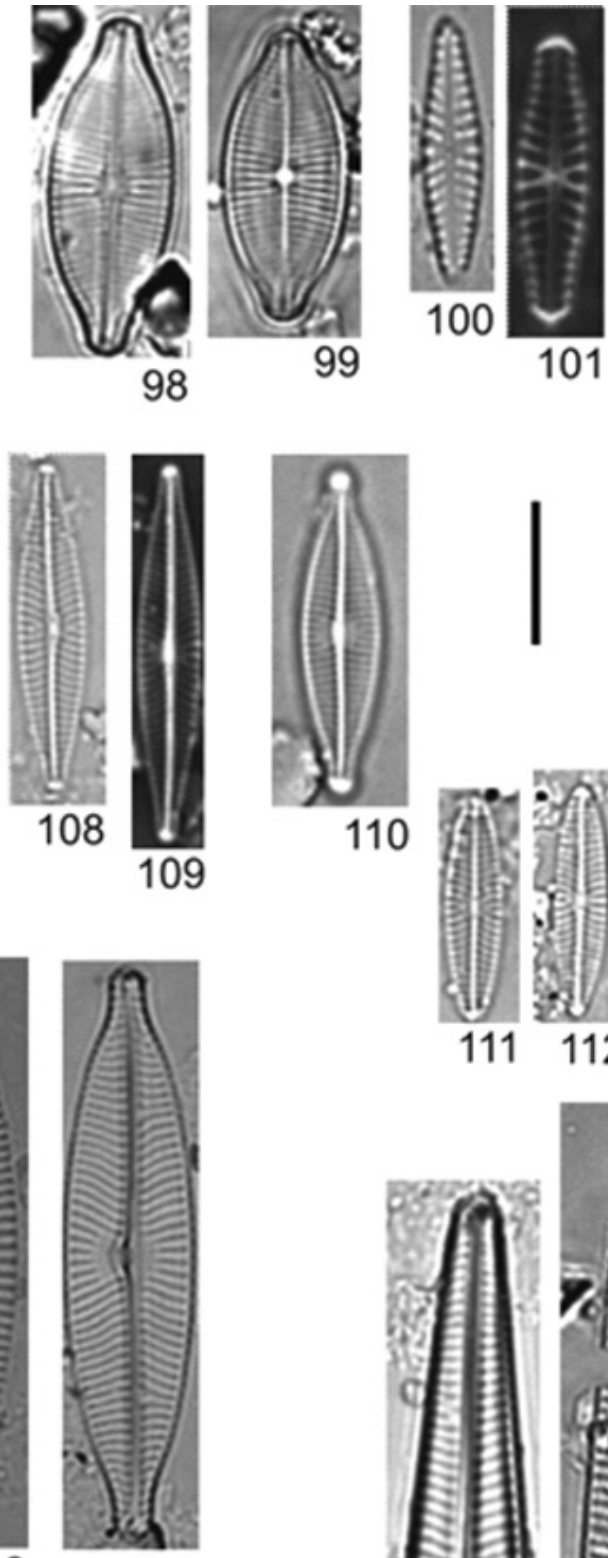

119

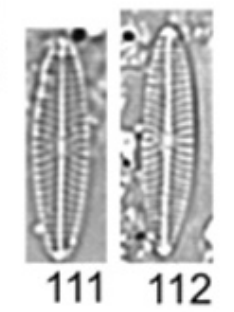

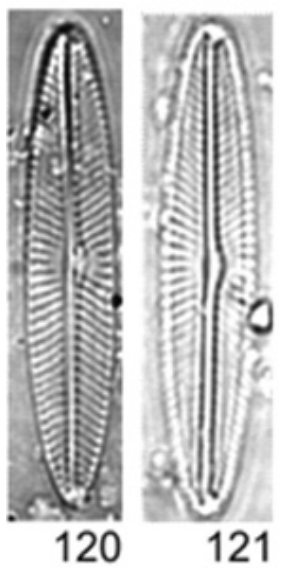
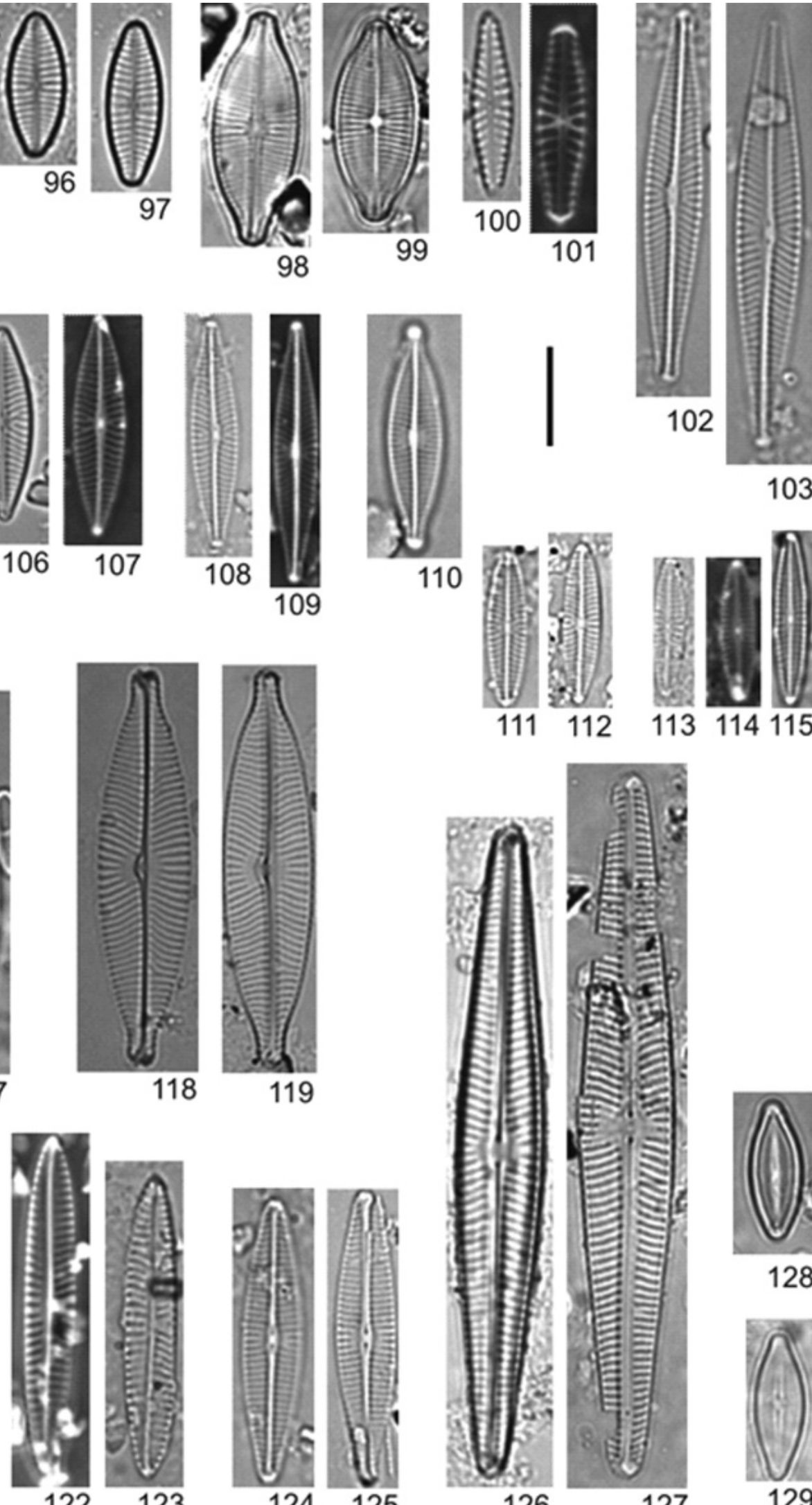

103
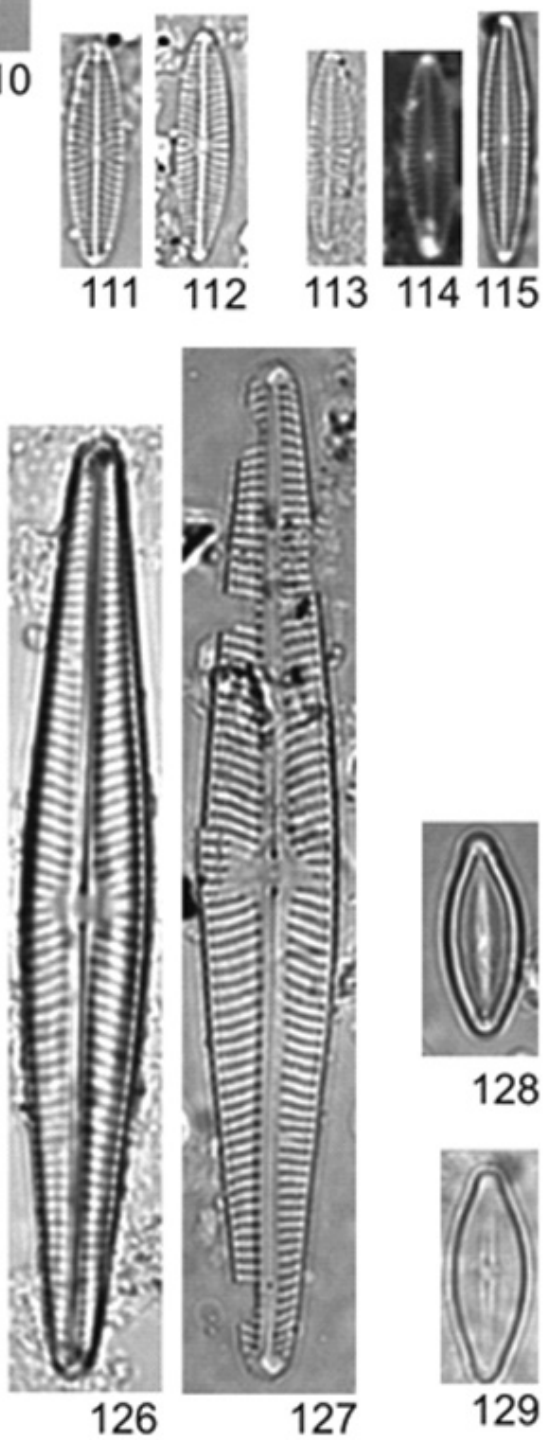

Figuras 94-129. Imagens em Microscópio Óptico. 94, 95. Geissleria aikenensis; 96, 97. Placogeia. kriegeri; 98, 99. Navigeia. lateropunctata; 100, 101. Hippodonta hungarica; 102, 103. Navicula leptostriata; 104, 105. N. cryptocephala; 106, 107. N. cryptotenella; 108, 109. N. notha; 110. N. gregaria; 111, 112. N. microdigitoradiata; 113-115. N. salinicola; 116, 117. N. viridula var. germainii; 118, 119. N. rostellata; 120, 121. . symmetrica; 122, 123. N. eichhorniaephila; 124, 125. N. namibica; 126, 127. N. tripuntata; 128, 129. Nupela pardinhoensis. Barras $=10 \mu \mathrm{m}$ 
Material examinado: FLOR 49737, 49743, 49748, 50144, 50853, 50854, 50855, 50856, 50858, 50859, 50860, 50864, 50865, 50866, 50867.

Referência: Patrick \& Reimer (1966).

\section{Navicula tripuctata (O.F. Müller) Bory}

(Figs. 126, 127)

Morfometria: compr. 56,2-70,0 $\mu \mathrm{m}$, larg. 9,7-10,0 $\mu \mathrm{m}$, 11-12 estrias em $10 \mu \mathrm{m}$

Material examinado: FLOR 49743, 49748.

Referências: Metzeltin \& García-Rodrigues (2003), Hofmann et al. (2011).

Navicula viridula var. germainii (J.H. Wallace) LangeBertalot

(Figs. 116, 117)

Morfometria: compr. 36,6-42,2 $\mu \mathrm{m}$, larg. 7,3-9,1 $1 \mu \mathrm{m}, 14-$ 15 estrias em $10 \mu \mathrm{m}$.

Material examinado: FLOR 49737, 49743, 49748, 50144, 50853, 50854, 50856, 50859, 50864, 50867.

Referências: Wallace (1960), Hofmann et al. (2011).

Familia incertae sedis

Navigeia Bukhtiyarova

Navigeia lateropunctata (Wallace) Bukhtiyarova, Bacillariophyta of Lake Baikal, p. 169. 2013.

(Figs. 98, 99)

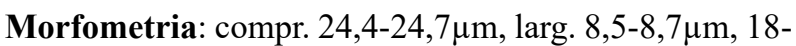
19 estrias em $10 \mu \mathrm{m}$.

Material examinado: FLOR 49737, 50144, 50853, 50854, 50856, 50859, 50864, 50865, 50866.

Referência: como Navicula lateropunctata Wallace (Patrick \& Reimer 1966).

Placogeia L. N. Bukhtiyarova

Placogeia kriegeri (Krasske) Bukhtiyarova in Bukhtiyarova \& Pomazkina Bacillariophyta of Lake Baikal, p. 170. 2013.

(Figs. 96, 97)

Morfometria: compr. 15,1-16,1 $\mu \mathrm{m}$, larg. 6,2-6,6 $6 \mathrm{~m}, 14-$ 16 estrias em $10 \mu \mathrm{m}$.

Material examinado: FLOR 50853.

Referências: como Navicula kriegeri Krasske (Krammer \& Lange-Bertalot 1986), Lange-Bertalot et al. (1996).
Brachysiraceae D.G. Mann

Nupela Vyvermann \& Compère

Nupela pardinhoensis Bes, Torgan \& Ector, Iheringia, Sér. Bot., 67(1): 93-125. 2012.

(Figs. 128, 129)

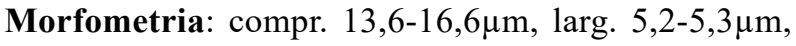
estrias inconspícuas.

Material examinado: FLOR 49737, 50853, 50854, 50859, 50862, 50865, 50866, 50867.

Referência: Bes et al. (2012).

As famílias mais representativas na Bacia Hidrográfica do Rio Itajaí foram Naviculaceae e Gomphonemataceae com 15 táxons cada. Os gêneros que apresentaram maior riqueza de espécies foram Gomphonema (15 táxons) e Navicula (13 táxons). Gomphonema adere-se ao substrato por pedúnculos gelatinosos, enquanto Navicula se mantem aderida à rocha por meio de mucilagem secretada pela rafe Wehr (2003), caracteristícas estas que favorecem o desenvolvimneto das espécies no epilíton.

Dentre os táxons identificados, 28 são primeiras citações para Santa Catarina, são elas: Achnanthidium atomus, $A$. pyrenaicum, Adlafia drouetiana, Cymbella excisa var angusta, Encyonema cf. neogracile var. tenuipunctata, E. neomesianum, Gomphonema incognitum, G. mexicanum, G. pseudoaugur, G. pumilum, G. salae, G. turris var. coarctata, Navicula eichhorniaephila, N. gregaria, $N$. leptostriata, N. microdigitoradiata, N. namibica, N. notha, N. salinicola, Navicula tripuctata, Nupela pardinhoensis, Placoneis gracilis, P. opportuna, Planothidium bagualensis, P. incuriatum, $P$. salvadorianum, Platessa oblongella, Psammothidium hustedtii.

Quanto à distribuição, os táxons mais frequentes, que ocorreram em $70 \%$ dos rios amostrados foram Achnanthidium atomus, A. exiguum, Encyonema silesiacum, Geissleria aikenesis, Gomphonema lagenula, Navicula cryptotenella, $N$. rostellata, $N$. symmetrica, Planothidium bagualensis e P. incuriatum (Tabela 2). Geissleria aikenensis e Gomphonema lagenula (citado como G. parvulum) foram também frequentes no Rio Itajaí-Mirim (Burliga et al. 2005, Sutil et al. 2007).

O número expressivo de táxons encontrados na Bacia Hidrográfica do Rio Itjaí e de citações pioneiras para o estado de Santa Catarina vem demonstrar a contribuição deste estudo ao conhecimento da biodiversidade de diatomáceas em ambientes lóticos no sul do Brasil. 
Tabela 2. Distribuição dos táxons de diatomáceas epilíticas pertencentes as famílias Cymbellaceae, Gomphonemaceae, Achnanthidiaceae, Brachysiraceae e Naviculaceae nos pontos amostrais na bacia hidrográfica do rio Itajaí.

\begin{tabular}{|c|c|c|c|c|c|c|c|c|c|c|c|c|c|c|c|c|c|c|c|c|}
\hline Táxons & 01 & 07 & 09 & 10 & 12 & 16 & 17 & 18 & 19 & 22 & 23 & 24 & 26 & 27 & 31 & 35 & 39 & 40 & 41 & 42 \\
\hline Achnanthidium atomus & $\mathrm{x}$ & $\mathrm{x}$ & $\mathrm{x}$ & $\mathrm{x}$ & $\mathrm{x}$ & $\mathrm{x}$ & $\mathrm{x}$ & - & $\mathrm{x}$ & $\mathrm{x}$ & $\mathrm{x}$ & $\mathrm{x}$ & - & $\mathrm{x}$ & - & $\mathrm{x}$ & $\mathrm{x}$ & $\mathrm{x}$ & $\mathrm{x}$ & $\mathrm{x}$ \\
\hline A. exiguum & $\mathrm{x}$ & - & $\mathrm{x}$ & $\mathrm{x}$ & $\mathrm{x}$ & $\mathrm{X}$ & $\mathrm{x}$ & $\mathrm{x}$ & $\mathrm{x}$ & $\mathrm{x}$ & $\mathrm{x}$ & $\mathrm{X}$ & - & $\mathrm{x}$ & $\mathrm{x}$ & - & $\mathrm{x}$ & $\mathrm{x}$ & $\mathrm{x}$ & $\mathrm{x}$ \\
\hline A. minutissimum & $\mathrm{x}$ & $\mathrm{x}$ & $\mathrm{x}$ & $\mathrm{x}$ & $\mathrm{x}$ & $\mathrm{x}$ & $\mathrm{x}$ & - & - & - & - & - & - & - & - & $\mathrm{x}$ & $\mathrm{x}$ & $\mathrm{x}$ & $\mathrm{x}$ & $\mathrm{x}$ \\
\hline A. pyrenaicum & $\mathrm{x}$ & - & - & - & $\mathrm{x}$ & - & - & - & - & - & - & - & - & - & - & $\mathrm{X}$ & $\mathrm{x}$ & - & $\mathrm{x}$ & - \\
\hline Adlafia drouetiana & - & $\mathrm{x}$ & - & - & - & $\mathrm{x}$ & $\mathrm{x}$ & $\mathrm{x}$ & $\mathrm{x}$ & $\mathrm{x}$ & $\mathrm{x}$ & - & - & - & - & $\mathrm{x}$ & - & $\mathrm{x}$ & $\mathrm{x}$ & $\mathrm{x}$ \\
\hline Capartogramma crucicula & $\mathrm{x}$ & - & - & $\mathrm{x}$ & $\mathrm{x}$ & $\mathrm{x}$ & - & $\mathrm{x}$ & - & - & - & - & - & - & - & - & - & - & - & - \\
\hline Cymbella excisa var. angusta & - & $\mathrm{x}$ & - & - & - & - & - & - & - & - & - & - & - & - & - & - & - & - & - & - \\
\hline C. tumida & - & $\mathrm{x}$ & - & - & - & - & - & - & - & - & - & $\mathrm{x}$ & - & - & - & $\mathrm{x}$ & - & $\mathrm{x}$ & - & - \\
\hline Cymbopleura amphicephala & - & - & - & - & $\mathrm{x}$ & - & - & - & - & - & - & - & - & - & - & - & - & - & - & - \\
\hline Encyonema minutum & $\mathrm{x}$ & - & - & $\mathrm{x}$ & $\mathrm{X}$ & - & - & - & $\mathrm{x}$ & $\mathrm{x}$ & $\mathrm{x}$ & $\mathrm{x}$ & - & - & - & $\mathrm{x}$ & $\mathrm{x}$ & $\mathrm{X}$ & $\mathrm{x}$ & - \\
\hline $\begin{array}{l}\text { E. cf. neogracile var. } \\
\text { tenuipunctata }\end{array}$ & - & - & - & - & - & - & - & - & - & - & - & - & $\mathrm{X}$ & - & - & - & - & - & - & - \\
\hline E. neomesianum & $\mathrm{x}$ & $\mathrm{x}$ & - & - & $\mathrm{x}$ & - & - & $\mathrm{x}$ & - & - & $\mathrm{x}$ & $\mathrm{x}$ & - & - & - & $\mathrm{x}$ & - & $\mathrm{x}$ & $\mathrm{x}$ & - \\
\hline E. perpusillum & - & - & $\mathrm{x}$ & - & $\mathrm{x}$ & $\mathrm{x}$ & $\mathrm{x}$ & $\mathrm{x}$ & $\mathrm{x}$ & $\mathrm{x}$ & $\mathrm{x}$ & $\mathrm{x}$ & $\mathrm{x}$ & $\mathrm{x}$ & - & - & - & $\mathrm{x}$ & $\mathrm{x}$ & - \\
\hline E. silesiacum & $\mathrm{x}$ & $\mathrm{X}$ & $\mathrm{X}$ & $\mathrm{X}$ & $\mathrm{x}$ & $\mathrm{X}$ & $\mathrm{X}$ & $\mathrm{X}$ & - & - & $\mathrm{x}$ & $\mathrm{X}$ & $\mathrm{X}$ & $x$ & $\mathrm{X}$ & $\mathrm{X}$ & $\mathrm{X}$ & $\mathrm{X}$ & $\mathrm{X}$ & - \\
\hline Encyonema sp. & - & - & - & - & $\mathrm{x}$ & - & - & - & - & - & - & $\mathrm{x}$ & - & - & - & - & - & - & - & - \\
\hline Geissleria aikenensis & $\mathrm{x}$ & $\mathrm{x}$ & $\mathrm{x}$ & $\mathrm{x}$ & $\mathrm{x}$ & $\mathrm{x}$ & $\mathrm{x}$ & $\mathrm{x}$ & $\mathrm{x}$ & $\mathrm{x}$ & $\mathrm{x}$ & $\mathrm{x}$ & - & $\mathrm{x}$ & - & $\mathrm{x}$ & $\mathrm{x}$ & $\mathrm{x}$ & $\mathrm{x}$ & $\mathrm{x}$ \\
\hline Gomphonema angustatum & - & - & - & $\mathrm{x}$ & $\mathrm{x}$ & - & - & $\mathrm{x}$ & - & - & - & - & - & - & - & - & - & - & $\mathrm{x}$ & - \\
\hline G. brasiliense ssp. pacificum & - & - & $\mathrm{x}$ & $\mathrm{x}$ & $\mathrm{x}$ & - & - & - & $\mathrm{x}$ & - & - & $\mathrm{x}$ & $\mathrm{x}$ & $\mathrm{x}$ & - & - & - & - & - & - \\
\hline G. brasiliensoide & - & - & $\mathrm{x}$ & $\mathrm{x}$ & $\mathrm{x}$ & - & - & - & $\mathrm{x}$ & - & - & $\mathrm{x}$ & $\mathrm{x}$ & $\mathrm{x}$ & - & - & - & - & - & - \\
\hline G. gracile & - & - & $\mathrm{x}$ & - & - & $\mathrm{x}$ & - & $\mathrm{x}$ & - & $\mathrm{x}$ & - & - & $\mathrm{x}$ & - & - & - & - & - & - & - \\
\hline G. incognitum & - & - & $\mathrm{x}$ & $\mathrm{x}$ & $\mathrm{x}$ & $\mathrm{x}$ & - & - & $\mathrm{X}$ & $\mathrm{x}$ & $\mathrm{x}$ & $\mathrm{x}$ & $\mathrm{x}$ & $\mathrm{x}$ & $\mathrm{x}$ & - & $\mathrm{x}$ & $\mathrm{x}$ & - & $\mathrm{x}$ \\
\hline G. lagenula & $\mathrm{x}$ & $\mathrm{x}$ & $\mathrm{x}$ & $\mathrm{x}$ & $\mathrm{x}$ & $\mathrm{x}$ & $\mathrm{x}$ & $\mathrm{x}$ & $\mathrm{x}$ & - & $\mathrm{x}$ & $\mathrm{x}$ & $\mathrm{x}$ & $\mathrm{x}$ & - & $\mathrm{x}$ & $\mathrm{x}$ & $\mathrm{x}$ & $\mathrm{x}$ & $\mathrm{x}$ \\
\hline G. mexicanum & - & - & $\mathrm{x}$ & - & - & - & - & - & - & - & - & - & - & $\mathrm{x}$ & - & - & - & - & - & - \\
\hline G. parvulum & $\mathrm{x}$ & $\mathrm{x}$ & $\mathrm{x}$ & $\mathrm{x}$ & $\mathrm{x}$ & - & - & $\mathrm{x}$ & $\mathrm{X}$ & $\mathrm{x}$ & $\mathrm{x}$ & - & - & - & - & $\mathrm{x}$ & $\mathrm{X}$ & $\mathrm{x}$ & $\mathrm{x}$ & - \\
\hline G. pseudoaugur & - & $\mathrm{x}$ & $\mathrm{x}$ & - & $\mathrm{x}$ & - & - & - & $\mathrm{x}$ & - & $\mathrm{x}$ & - & - & - & - & - & - & - & $\mathrm{x}$ & - \\
\hline G. pumilum & - & $\mathrm{x}$ & $\mathrm{x}$ & - & - & $\mathrm{x}$ & - & $\mathrm{x}$ & - & - & - & - & - & $\mathrm{x}$ & - & - & - & - & - & $\mathrm{x}$ \\
\hline G. rochense & - & - & - & - & - & - & - & - & $\mathrm{x}$ & - & - & - & - & - & - & - & - & - & - & - \\
\hline G. salae & - & - & - & - & $\mathrm{x}$ & - & - & - & - & - & - & - & - & - & - & - & - & - & - & - \\
\hline G. turris var. coarctata & - & - & - & - & - & - & - & - & - & - & - & - & $\mathrm{x}$ & - & - & - & - & - & - & - \\
\hline Gomphonema sp.1 & - & - & - & - & $\mathrm{x}$ & - & - & $\mathrm{x}$ & - & - & - & - & - & - & - & - & - & - & $\mathrm{x}$ & - \\
\hline Gomphonema sp. 2 & - & - & - & - & - & - & - & $\mathrm{x}$ & - & - & - & - & - & - & - & - & - & - & - & - \\
\hline Hippodonta hungarica & $\mathrm{x}$ & - & - & $\mathrm{x}$ & $\mathrm{x}$ & - & - & - & - & - & - & - & - & - & - & - & - & - & - & - \\
\hline Lemnicola hungarica & - & - & - & - & - & - & - & - & $\mathrm{x}$ & - & $\mathrm{x}$ & - & - & - & - & - & - & $\mathrm{x}$ & $\mathrm{x}$ & - \\
\hline Navicula cryptocephala & $\mathrm{x}$ & - & $\mathrm{x}$ & - & - & - & - & $\mathrm{x}$ & - & - & - & - & - & - & - & - & - & $\mathrm{x}$ & - & - \\
\hline N. cryptotenella & $\mathrm{x}$ & $\mathrm{x}$ & $\mathrm{x}$ & $\mathrm{x}$ & $\mathrm{x}$ & $\mathrm{x}$ & $\mathrm{x}$ & - & $\mathrm{x}$ & $\mathrm{x}$ & $\mathrm{x}$ & - & - & - & - & $\mathrm{x}$ & - & $\mathrm{x}$ & $\mathrm{x}$ & $\mathrm{x}$ \\
\hline N. eichhorniaephila & - & - & - & - & - & - & - & $\mathrm{x}$ & - & - & - & - & - & - & - & - & - & - & - & - \\
\hline N. gregaria & - & - & $\mathrm{x}$ & - & - & $\mathrm{X}$ & - & $\mathrm{x}$ & $\mathrm{x}$ & $\mathrm{x}$ & - & - & - & - & - & - & - & $\mathrm{x}$ & $\mathrm{x}$ & $\mathrm{x}$ \\
\hline N. leptostriata & - & - & - & - & $\mathrm{x}$ & - & - & - & - & - & $\mathrm{x}$ & - & - & - & - & - & $\mathrm{x}$ & - & - & - \\
\hline N. microdigitoradiata & - & - & $\mathrm{x}$ & - & $\mathrm{x}$ & - & - & - & - & - & $\mathrm{x}$ & - & - & - & - & - & - & - & $\mathrm{x}$ & - \\
\hline N. namibica & - & - & - & - & $\mathrm{x}$ & - & - & - & - & - & $\mathrm{x}$ & - & - & - & - & - & - & - & - & - \\
\hline N. notha & $\mathrm{x}$ & $\mathrm{x}$ & $\mathrm{x}$ & $\mathrm{x}$ & $\mathrm{x}$ & - & - & - & $\mathrm{x}$ & $\mathrm{x}$ & $\mathrm{x}$ & - & - & - & - & $\mathrm{x}$ & - & $\mathrm{x}$ & $\mathrm{x}$ & - \\
\hline N. rostellata & - & $\mathrm{x}$ & $\mathrm{x}$ & $\mathrm{x}$ & $\mathrm{x}$ & $\mathrm{x}$ & $\mathrm{x}$ & $\mathrm{x}$ & $\mathrm{x}$ & $\mathrm{x}$ & $\mathrm{x}$ & - & - & $\mathrm{x}$ & - & $\mathrm{x}$ & $\mathrm{x}$ & $\mathrm{x}$ & $\mathrm{x}$ & - \\
\hline N. salinicola & - & - & - & - & $\mathrm{x}$ & - & - & - & - & - & - & - & - & - & - & - & - & - & - & - \\
\hline N. symmetrica & $\mathrm{x}$ & $\mathrm{x}$ & $\mathrm{x}$ & $\mathrm{x}$ & $\mathrm{x}$ & $\mathrm{X}$ & $\mathrm{x}$ & $\mathrm{x}$ & - & $\mathrm{x}$ & $\mathrm{x}$ & $\mathrm{x}$ & - & - & - & $\mathrm{x}$ & $\mathrm{x}$ & $\mathrm{x}$ & $\mathrm{x}$ & - \\
\hline N. viridula var. germainii & $\mathrm{x}$ & $\mathrm{x}$ & $\mathrm{x}$ & $\mathrm{X}$ & $\mathrm{X}$ & $\mathrm{X}$ & - & $\mathrm{X}$ & - & - & $\mathrm{X}$ & - & - & - & - & $\mathrm{X}$ & - & - & $\mathrm{x}$ & - \\
\hline N. tripunctata & - & $\mathrm{x}$ & $\mathrm{x}$ & - & - & - & - & - & - & - & - & - & - & - & - & - & - & - & - & - \\
\hline
\end{tabular}


Tabela 2. Cont.

\begin{tabular}{|c|c|c|c|c|c|c|c|c|c|c|c|c|c|c|c|c|c|c|c|c|}
\hline Táxons & 01 & 07 & 09 & 10 & 12 & 16 & 17 & 18 & 19 & 22 & 23 & 24 & 26 & 27 & 31 & 35 & 39 & 40 & 41 & 42 \\
\hline Navigeia lateropunctata & $\mathrm{x}$ & - & - & $\mathrm{x}$ & $\mathrm{x}$ & $\mathrm{x}$ & - & $\mathrm{x}$ & - & - & $\mathrm{x}$ & - & - & - & - & $\mathrm{x}$ & $\mathrm{x}$ & $\mathrm{x}$ & - & - \\
\hline Nupela pardinhoensis & $\mathrm{x}$ & - & - & - & $\mathrm{x}$ & $\mathrm{x}$ & - & - & - & - & $\mathrm{x}$ & - & - & $\mathrm{x}$ & - & - & $\mathrm{x}$ & $\mathrm{x}$ & $\mathrm{x}$ & - \\
\hline Placoneis gracilis & - & - & - & - & $\mathrm{x}$ & - & - & - & - & - & - & - & - & - & - & - & - & - & - & - \\
\hline P. madagascariense & - & - & - & $\mathrm{x}$ & - & - & - & - & - & - & - & - & - & $\mathrm{x}$ & - & - & - & - & - & - \\
\hline P. opportuna & - & - & - & - & $\mathrm{x}$ & - & - & - & - & - & - & - & - & - & - & - & - & - & - & - \\
\hline$P$. porifera & - & - & - & - & - & - & - & - & $\mathrm{x}$ & - & - & - & - & - & $\mathrm{x}$ & - & - & $\mathrm{x}$ & $\mathrm{x}$ & - \\
\hline Placogeia kriegeri & - & - & - & - & $\mathrm{x}$ & - & - & - & - & - & - & - & - & - & - & - & - & - & - & - \\
\hline Planothidium bagualensis & $\mathrm{x}$ & - & $\mathrm{x}$ & $\mathrm{x}$ & $\mathrm{x}$ & $\mathrm{x}$ & $\mathrm{x}$ & - & $\mathrm{x}$ & - & $\mathrm{x}$ & $\mathrm{x}$ & $\mathrm{x}$ & - & $\mathrm{x}$ & - & $\mathrm{x}$ & $\mathrm{x}$ & $\mathrm{x}$ & - \\
\hline P. heteromorphum & - & - & - & - & - & - & $\mathrm{x}$ & - & - & - & - & - & - & - & - & - & - & - & - & - \\
\hline P. incuriatum & $\mathrm{x}$ & $\mathrm{x}$ & $\mathrm{x}$ & $\mathrm{x}$ & - & $\mathrm{x}$ & $\mathrm{x}$ & $\mathrm{x}$ & $\mathrm{x}$ & $\mathrm{x}$ & $\mathrm{x}$ & $\mathrm{x}$ & - & $\mathrm{x}$ & - & $\mathrm{x}$ & $\mathrm{x}$ & $\mathrm{x}$ & $\mathrm{x}$ & - \\
\hline P. salvadorianum & - & - & - & - & - & - & - & - & - & - & - & - & - & - & - & - & $\mathrm{x}$ & - & - & - \\
\hline Platessa oblongella & - & - & - & - & - & - & - & - & - & $\mathrm{x}$ & - & - & - & - & - & - & $\mathrm{x}$ & - & - & - \\
\hline Psammothidium hustedtii & $\mathrm{x}$ & - & - & - & $\mathrm{x}$ & - & - & - & $\mathrm{x}$ & - & - & - & - & - & - & - & - & - & $\mathrm{x}$ & - \\
\hline
\end{tabular}

\section{AGRADECIMENTOS}

Agradecemos ao Conselho Nacional de Desenvolvimento Científico e Tecnológico, à Fundação de Amparo à Pesquisa e Inovação do Estado de Santa Catarina, à EcoAquatica P. D. C. A., e ao Fundo Setorial de Recursos Hídricos pelo apoio estrutural e financeiro no desenvolvimento do projeto "Delineamento de Ecorregiões Aquáticas - Costa Sudeste" (Edital CT Hidro/CNPq 37/2005 Processo 557352/20053). Ao Laboratório Central de Microscopia Eletrônica da Universidade Federal de Santa Catarina, pela permissão de uso do microscópio eletrônico de varredura.

\section{REFERÊNCIAS}

Allan, J.D.S. \& Castillo, M.M. 2007. Stream Ecology, structure and function of running wates. Springer, Netherlands. 436p.

Araújo, N.B. de, Souza-Mosimann, R.M. de \& Fernandes, L. F. 1989. Contribuição ao estudo das diatomáceas (Bacillariophyceae) da Foz do Rio Araranguá, Santa Catarina, Brasil. Insula (19):305-332.

Bartozek, E.C.R, Bueno, N.C., Ludwig, T.A.V, Tremarin, P.I., Nardelli, M.S. \& Rocha A.C.R. 2013. Diatoms (Bacillariophyceae) of Iguaçu National Park, Foz do Iguaçu, Brazil. Acta Botanica Brasilica 27(1):108-123

Bellinger, E.G. \& Singee, D.C. 2010. Freshwater algae: Identification and Use as Bioindicators. Wiley-Blackwell, UK. 271p.

Bes, D., Ector, L., Torgan, L.C. \& Lobo, E.A. 2012. Composition of the epilithic diatom flora a subtropical river, Southern, Brazil. Iheringia. Série Botânica 67(1):93-125.

Burliga, A.L., Torgan, L.C., Nobrega, E.A, Beaumord, A., Costa, C.O. \& Yamauti, D.V. 2005. Diatomáceas epilíticas do rio Itajaí-Mirim, Santa Catarina, Brasil. Acta Scientiarum. Biological Sciences 27(4):415-421.

Butcher R.W. 1932. Studies in the ecology of rivers. II. The microflora of rivers with special reference to the algae on the river bed. Annals of Botany 46(184):813-861.

Chiossi, P. 2013. Composição e Estrutura das Assembleias de Diatomáceas Epilíticas e sua relação com a integridade ambiental de rios da Bacia Hidrográfica do Rio Itajaí, Santa Catarina. Dissertação 161p. Universidade Federal de Santa Catarina, Santa Catarina.

Chudaev, D.A. \& Gololobova, M.A. 2016. Diatoms of the Glubokoe lake (Moscow region). Moscou, Russia. p. 1-446.

Felício-Fernandes, G. 1996. Diatomáceas no Rio Tavares, Manguezal do Rio Tavares, Florianópolis, Santa Catarina, Brasil. II. Penatae. Insula (25):69-142.
Felício-Fernandes, G, Souza-Mosimann, R. \& Moreira Filho, H. 1994. Diatomáceas no Rio Tavares, Florianópolis, Santa Catarina, Brasil - I. Ordem Centrales (excluídas as famílias Rhizosoleniaceae e Chaetoceraceae). Insula (23):35-90.

Fernandes, L.F., Souza-Mosimann, R.M. de \& Felício-Fernandes, G., 1990. Diatomáceas (Bacillariophyceae) do Rio Ratones, Florianópolis, Santa Catarina, Brasil: I. Baixo Curso e Estuário. Insula (20):11-112.

Ferrari, F \& Ludwig, T.A.V. 2007. Coscinodiscophyceae, Fragilariophyceae e Bacillariophyceae (Achnanthales) dos rios Ivaí, São João e dos Patos, bacia hidrográfica do rio Ivaí, município de Prudentópolis, PR, Brasil. Acta Botanica Brasilica 21(2):421-441.

Hofmann, G., Werum, M. \& Lange-Bertalot, H. 2011. Diatomeen im Süßwasser-Benthos von Mitteleuropa. Bestimmungsflora Kieselalgen für die ökologische Praxis. Über 700 der häufigsten Arten und ihre Ökologie. Koeltz Scientific Books, Königstein. 908 p.

Hustedt, F. 1939. Die Diatomeenflora des Küstengebietes der Nordsee vom Dollart bis zur Elbemündung. I. Die Diatomeenflora in den Sedimenten der unteren Ems sowie auf den Watten in der Leybucht, des Memmert und bei der Insel Juist. Adhandlungen des Naturwissenschaftlichen Verein zu Bremen 31(2/3):571- 677.

Hustedt, F. 1944. Neue und wenig bekannte Diatomeen. Berichte der Deutschen Botanischen Gesellschaft 61:227-290.

Jüttner, I. E. Reichardt \& E.J. Cox. 2004. Taxonomy and ecology of some new Gomphonema species common in Himalayan streams. Diatom Research 19:235-264.

Kociolek, J.P. \& Kingston, J.C. 1999. Taxonomy, ultrastructure, and distribution of some gomphonemoid diatoms (Bacillariophyceae: Gomphonemataceae) from rivers in the United States. Canadian Journal of Botany 77:686-705.

Krammer, K. 1997. Die Cymbelloiden Diatomeen. Eine Monographie der weltweit bekannten taxa. Allgemeines und Encyonema Part. Bibliotheca Diatomologica 36:1-382.

Krammer, K. 2002. Cymbella. Diatoms of the European Inland Waters and Comparable Habitats. Diatoms of Europe 3:1- 584.

Krammer, K. 2003. Cymbopleura, Delicata, Navicymbula, Gomphocymbellopsis, Afrocymbella. Diatoms of the European Inland Waters and Comparable Habitats. Diatoms of Europe 4:1-530

Krammer, K. \& Lange-Bertalot, H. 1986. Bacillariophyceae: Naviculaceae. In Süßwasserflora von Mitteleuropa (H. Ettl et al., eds.). Gustav Fisher Verlag, Stuttgart, v. 2, n. 1, p. 1-876.

Krammer, K. \& Lange-bertalot, H. 1991. Bacillariophyceae: Achnanthaceae. Kritische Ergänzungen zu Navicula (Lineolatae) und Gomphonema. In Sübwasserflora von Mitteleuropa (H. Ettl et al., eds). Gustav Fisher Verlag, Stuttgart, v.2, n. 4, p 1-437.

Lange-Bertalot, H. 1993. 85 neue Taxa und über 100 weitere neu definierte Taxa ergänzend zur Süsswasserflora von Mitteleuropa. Bibliotheca Diatomologica 27:1-164 
Lange-Bertalot, H., Külbs, K., Lauser. T. Nörpel-Schempp, M. \& Willmann, M. 1996. Diatom Taxa introduced gy Georg Krasske Documnetation and Revison. Koeltz Scientific Books, Könongtein, $358 \mathrm{p}$.

Lange-bertalot, H. \& Metzeltin, D. 1996. Oligotrophie indikatoren. 800 taxa repräsentativ für drei diverse see-typen, kalkreich - oligodystroph - schwach gepuffertes weichwasser. Iconographia Diatomologica 2:1-390.

Lange-Bertalot, H., Metzeltin, D. \& Witkowski, A 1996. Hippodonta gen. nov. Umschreibung und Begründung einer neuer Gattung der Naviculaceae. Iconographia Diatomologica 4:247-275.

Medeiros, G., Amaral, M.W.W., Ferreira, P.C., Ludwig, T.V. \& Bueno, N.C. 2018. Gomphonema Ehrenberg (Bacillariophyceae, Gomphonemataceae) of the São Francisco Falso River, Paraná, Brazil Biota Neotropica 18(3):1-23

Medlin, L.K \& Kaczmarska, I. 2004. Evolution of the diatoms: V. Morphological and ecological support for the major clades and a taxonomic revision. Phycologia 43(3): 245-270.

Metzeltin, D. \& Lange-Bertalot, H. 1998. Tropical diatoms of the South America I. Iconografia Diatomologica 5:1-695.

Metzeltin, D. \& Lange-Bertalot, H. 2002. Diatoms from the "Island Constinent' Madagascar. Iconographia Diatomologica 11:7-286.

Metzeltin, D. \& García-Rodriguez, F. 2003. Las Diatomeas Uruguayas. D.I.R.A.C., Facultad de Ciencias, Montevideo. 207 p.

Metzeltin, D., Lang-Bertalot, H. \& García-Rodríguez 2005. Diatoms of Uruguay. Iconographia Diatomologica 15:9-736.

Monnier, O., Lange-Bertalot, H., Rimet, F., Hoffmann, L. \& Ector, L. 2004. Achnanthidium atomoides sp. nov., a new diatom from the Grand-Duchy of Luxembourg. Vie et Mileu 54:127-136.

Moreira-Filho, H. \& Valente-Moreira, I.M. 1981. Avaliação taxonômica e ecológica das diatomáceas (Bacillariophyceae) epífitas em algas pluricelulares obtidas nos litorais dos estados do Paraná, Santa Catarina e São Paulo. Boletim do Museu Botânico Municipal 47:1-17.

Moser, G. Lange-Bertalot, H. \& Metzeltin, D. 1998. Insel der Endemitten Geobotanisches Phänomen Neukaledonien. Bibliotheca Diatomologica 38:7-455.

Ohtsuka, T. \& Fujita, Y. 2001. The diatom flora and its seasonal changes in a paddy field in central Japan. Nova Hedwigia 73(1/2): 97-128.

Patrick, R. \& Reimer, C.W. 1966. The diatoms of the United States. Exclusive of Alaska and Hawaii. Fragilariaceae, Eunotiaceae, Achnanthaceae, Naviculaceae. Monographs of the Academy of Natural Sciences of Philadelphia, Philadelphia, v.1. 688 p.

Patrick, R. \& Reimer, C.W. 1975. The diatoms of the United States. Exclusive of Alaska and Hawaii. Academy of Natural Sciences of Philadelphia, Philadelphia, v. 2. 213p.

Potapova, M. 2010. Achanthidium exiguum. In Diatoms of North America. Disponível em: https://diatoms.org/species/achnanthidium_exiguum. Acessado em 06.07.2020.

Potapova, M. \& Hamilton, P.B.J. 2007. Morphological and ecological variation within the Achnanthidium minutissimum (Bacillariophyceae) species complex. Journal of Phycology 43:561-575.

Potapova, M.G. \& Ponader, K.C. 2004. Two common North American diatoms, Achnanthidium rivulare sp. nov. and A. deflexum (Reimer) Kingston: morphology, ecology and comparison with related species. Diatom Research 19:33-57.

Reichardt, E. 1999. Zur Revision der Gattung Gomphonema. Iconographia Diatomologica 8:5-203.

Reichard, E. 2005. Die Identität von Gomphonema entolejum Östrup (Bacillariophyceae) sowie Revision ähnliger Arten mit weiter Axialarea. Nova Hedwigia 8(1-2):115-144.

Reichardt, E. \& Lange-Bertalot, H. 1991. Taxonomische Revision des Artencomplexes um Gomphonema angustum - G. dichotomum - G. intricatum - G. vibrio und ahnliche Taxa (Bacillariophyceae). Nova Hedwigia 53(3-4):519-544.

Rodrigues, L. 1984. Contribuição ao conhecimento das diatomáceas do rio Tubarão - Santa Catarina - Brasil. Insula 14:47-120.

Rodrigues, L. 1988. Uma nova variedade para Cymbella perpusilla Cleve. Insula 18:99-103.
Rodrigues, L. 1991/1992. Diatomoflorula do Rio Tubarão, estado de Santa Catarina, Brasil: II. Naviculaceae Kützing. Insula 21:3-42.

Rodrigues, L. \& Moreira-Filho, H. 1990. Diatomoflórula do rio Tubarão, Santa Catarina, Brasil: I - Eunotiaceae Kützing e Achnanthaceae Kützing. Insula 20:113-135.

Ross, R. 1963. The diatom genus Capartogramma and the identity of Schizostauron. Bulletin of the British Museum. Botany Series 3(2): 49-92.

Round, F.E. \& Basson, P.W. 1997. A new monoraphid diatom genus (Pogoneis) from Bahrain and the transfer of previously described species A. hungarica and A. taeniata to new genera. Diatom Research 12(1):71-81.

Round, F.E., Crawford, R.M. \& Mann, D.G. 1990. The Diatoms: Biology and Morphology of the Genera. Cambridge University Press, Cambridge. 747 p.

Rumrich, U., Lange-Bertalot, H. \& Rumrich, M. 2000. Diatoms of the Andes. From Venezuela to Patagonia/Tierra del Fuego and two additional contributions. Iconographia Diatomologica 9:1-637.

Sar, E.A., Sala, S.E., Sunesen, I., Henninger, M.S. \& Montastruc, M. 2009. Catálogo de los géneros, especies y taxa infraespecíficos erigidos por J. Frenguelli. Diatom Monographs 10:1-419.

Silva, J.F., Salomoni, S. E., Oliveira, M.A. \& Machado. S.L.O. 2017. Diatomáceas epilíticas de sistemas lóticos na sub-bacia hidrográfica do Arroio Grande, Rio Grande do Sul, Brasil. Iheringia. Série Botânica 72(2):201-219.

Simonsen, R. 1974. The diatom plankton of the Indian Ocean expedition of R/V “Meteor", 1964-1965. Meteor. Forschungsergebnisse Reihe D-Biologie 19:1-66.

Stevenson, R.J. 1996. An Introduction to Algal Ecology in Freshwater Bentic Habitats. In Algal Ecology: Freshwater Benthic Eecosystems (R.J. Stevenson, M.L. Bothwell \& R.L. Lowe, eds.). Academic Press, San Diego, p. 3-26.

Stevenson, R. \& Pan, Y. 1999. Assessing environmental conditions in rivers and streams with diatoms. In The Diatoms: applications for the environmental and earth sciences (E. F. Stoermer \& J.O. Smol, eds.). Cambridge University Press, Cambridge p.11-40.

Sutil, C., Burliga, A.L., Beaumord, A.C. \& Scheffer, R. 2007. Composição e distribuição espacial das assembléias de diatomáceas epilíticas do Rio Itajaí-Mirim, Santa Catarina. In Anais do VIII Congresso de Ecologia do Brasil. Sociedade de Ecologia do Brasil, Caxambu, p. 1-3.

Torgan, L.C. \& Oliveira, M.A. 2001. Geissleria aikenensis (Patrick) Torgan et Oliveira comb. nov.: Morphological and ecological characteristics. In Proceedings of $16^{\text {th }}$ International Diatom Symposium (A. Economou-Amilli, ed.). Amvrosiou Press, Athens, p. $115-125$.

Tremarin, P.I., Ludwig, T.A.V., Bertolli, L.M., Faria, D.M. \& Costin, J.C. 2009. Gomphonema Ehrenberg e Gomphosphenia Lange-Bertalot (Bacillariophyceae) do Rio Maurício, Paraná, Brasil. Biota Neotropica 9(4):111-130.

Van Heurck H. 1880-1881. Synopsis des Diatomées de Belgique Atlas. Pls. I-XXX. Edite par LÁuteus, Anvers.

Wallace, J. 1960. New and variable diatoms. Notulae Naturae 331:1-8.

Wehr, J.D. 2003. Freshwater habitats of algae In Freshwater Algae of North America. Ecologia e Classification (J.D. Wehr \& R.G. Sheath, eds.). Academic Press, California, p. 11-45.

Wetzel, C.E. \& Ector, L. 2014. Taxonomy, distribution and autecology of Planothidium bagualensis sp. nov. (Bacillariophyta) a commom monoraphid species from southern Brazil rivers. Phytotaxa 156(4):201-210.

Wetzel C.E., Lange-Bertalot H. \& Ector E. 2017. Type analysis of Achnanthes oblongella Østrup and resurrection of Achnanthes saxonica Krasske (Bacillariophyta). Nova Hedwigia, Beiheft 146:209-227.

Wetzel, C.E., Vijver, B.V., Hofmann, L. \& Ector, L. 2013. Planothidium incuriatum sp. nov. a widely distributed diatom species (Bacillariophyta) and type analysis of Planothidium biporomum. Phytotaxa 138(1):43-57. 